\title{
Nonradial and nonpolytropic astrophysical outflows
}

\section{Relativistic MHD rotating spine jets in Kerr metric}

\author{
L. Chantry ${ }^{1}$, V. Cayatte ${ }^{1}$, C. Sauty ${ }^{1}$, N. Vlahakis ${ }^{2}$, and K. Tsinganos ${ }^{2}$ \\ ${ }^{1}$ Laboratoire Univers et Théories, Observatoire de Paris - PSL, UMR 8102 du CNRS, Université Paris Diderot, 92190 Meudon, France \\ e-mail: loic.chantry@obspm.fr \\ ${ }^{2}$ Section of Astrophysics, Astronomy and Mechanics, Department of Physics, University of Athens, Panepistimiopolis Zografos, \\ Athens 15783, Greece \\ e-mail: christophe.sauty@obspm.fr
}

Received 17 August 2017 / Accepted 15 December 2017

\begin{abstract}
Context. High-resolution radio imaging of active galactic nuclei (AGN) has revealed that the jets of some sources present superluminal knots and transverse stratification. Recent observational projects, such as ALMA and $\gamma$-ray telescopes, such as HESS and HESS2 have provided new observational constraints on the central regions of rotating black holes in AGN, suggesting that there is an inneror spine-jet surrounded by a disk wind. This relativistic spine-jet is likely to be composed of electron-positron pairs extracting energy from the black hole and will be explored by the future $\gamma$-ray telescope CTA.

Aims. In this article we present an extension to and generalization of relativistic jets in Kerr metric of the Newtonian meridional selfsimilar mechanism. We aim at modeling the inner spine-jet of AGN as a relativistic light outflow emerging from a spherical corona surrounding a Kerr black hole and its inner accretion disk.

Methods. The model is built by expanding the metric and the forces with colatitude to first order in the magnetic flux function. As a result of the expansion, all colatitudinal variations of the physical quantities are quantified by a unique parameter. Unlike previous models, effects of the light cylinder are not neglected.

Results. Solutions with high Lorentz factors are obtained and provide spine-jet models up to the polar axis. As in previous publications, we calculate the magnetic collimation efficiency parameter, which measures the variation of the available energy across the field lines. This collimation efficiency is an integral part of the model, generalizing the classical magnetic rotator efficiency criterion to Kerr metric. We study the variation of the magnetic efficiency and acceleration with the spin of the black hole and show their high sensitivity to this integral.

Conclusions. These new solutions model collimated or radial, relativistic or ultra-relativistic outflows in AGN or $\gamma$-ray bursts. In particular, we discuss the relevance of our solutions to modeling the M 87 spine-jet. We study the efficiency of the central black hole spin to collimate a spine-jet and show that the jet power is of the same order as that determined by numerical simulations.
\end{abstract}

Key words. black hole physics - magnetohydrodynamics (MHD) - relativistic processes - galaxies: jets

\section{Introduction}

Active galactic nuclei (AGN) jets are now recognized as being multi-component outflows related to accretion onto a supermassive black hole (SMBH). The outer kiloparsec/megaparsec-scale lobes are fed by a powerful hadronic plasma, which most likely originates from the Keplerian accretion disk via the magnetocentrifugal launching mechanism, Blandford \& Payne (1982). This hadronic population could be responsible for the second component peaking in the $\gamma$-ray band of the spectral energy distribution (SED) of some blazars. As explained in Böttcher et al. (2013), this is possible if a relativistic jet of protons contributes significantly to the radiative output through proton synchrotron emission or photo-pion production. However the purely hadronic synchrotron models for blazars present a major problem due to the requirement for having very high powers in the jets. Indeed, hadronic processes are very inefficient (Sikora 2011). They are only energetically favorable for high-frequency synchrotron peak (HSP) blazars, where the jet is likely to be highly magnetized (Petropoulou \& Dermer 2016).
The alternative approach to explaining the origin of the high-energy emission is to consider leptonic models. There, the radiative output throughout the electromagnetic spectrum is assumed to be dominated by leptons both at low and high frequencies. Inverse Compton scattering of soft photons (IC) by relativistic nonthermal electrons in the jet is supposed to be the most probable mechanism for $\gamma$-ray production, at least for strong-line and low-frequency synchrotron peak - LSP blazars. Until recently, leptonic models in quasi steady-state were very successful in modeling the SED for almost all classes of blazars (e.g., Celotti \& Ghisellini 2008). However leptonic models with a one-zone component where only one portion of the jet dominates the emission are now questioned. Mostly, they fail to produce the extremely high bulk Lorentz factors in a very compact emission region required for ultra-fast variability of some TeV blazars (Begelman et al. 2008). Despite several proposed mechanisms mostly based on small-scale inhomogeneities in the jet (see references in Vovk \& Babić 2015), it is not yet possible to know the location of the variable $\gamma$-ray emission; it may be at the base of the jet or up to 
parsec-scale distances from the central black hole. Moreover most of the sources in which ultra-rapid variability events have been observed are BL Lac objects emitting very-high-energy radiation

For BL Lacs the application of the one-zone synchrotronself Compton model to the SED implies that the jet is weakly magnetized and the emitting region is far from equipartition. Both conditions are required if magnetic-reconnection-powered emission is at the origin of the ultra-rapid variability (Tavecchio 2016). A most important constraint comes from the launching and the acceleration processes, which should lead to almost equipartition between the magnetic and the kinetic energy fluxes as can be seen in other blazars. In order to unify the BL Lac and radiogalaxy populations, Ghisellini et al. (2005) already proposed a structured jet model with two components; a faster core (the spine) surrounded by a slower sheath or layer. This two component model is able to explain peculiar features of $\mathrm{TeV}$-emitting BL Lacs, such as the absence of fast superluminal components and the presence of a limb-brightened radio structure (Giroletti et al. 2004; Nagai et al. 2014). Tavecchio \& Ghisellini (2016) showed that this structured jet model gives an extra source of soft photons intervening in the IC emission for BL Lacs. It is due to the radiative interplay between the two components and allows to reproduce the emission in equipartition conditions. This spinesheath jet structure has also been explored in Sikora et al. (2016) for strong-line blazars in order to explain why $\gamma$-ray variations are often observed to have much larger amplitudes than the corresponding optical variations, as well as other observed features of $\gamma$-ray flares. For the nearby radiogalaxy M 87, a direct detection of different motions was possible and an extended mildly relativistic flow is seen surrounding a relativistic central jet (Mertens et al. 2016).

The possible seed photon population at the origin of IC scattering in AGNs is questionable. The dominant seed source depends critically on the location of the primary emitting region in the jet (Finke 2016). Radio array observations of individual blazars are used to localize the $\gamma$-ray emission site among the superluminal components seen along the jet. However, they cannot put strong constraints on localizing the emission regions of the high-energy flares. More theoretical and numerical modelings and carefully analyzed polarization data are needed (see Kravchenko et al. 2016 and references therein). We note also the treatment used in Hada et al. (2011) for locating the central black hole relatively close to the jet base-radio core in M 87 using the dependence of the core position on frequency. There are cases where the spine-sheath model should work. The leptons in superluminal components after leaving the black hole corona interact with the sheath where IC scattering of photons occurs and produce the observed $\gamma$-ray activity (Marscher et al. 2010; Casadio et al. 2015).

For AGN jets, two-component models have been theoretically studied in Sol et al. (1989) and further developed by several authors (e.g., Fabian \& Rees 1995). In those studies the spine jet is the inner leptonic component (electron-positron pairs), which is self collimated inside a hadronic disk wind, and should be separated from the wind by a force-free, empty region. On the other hand, it has been shown numerically that the high collimation efficiency of the outer-disk wind is sufficient to prevent decollimation from the inertia of the inner relativistic plasma, in the case where the initial magnetic field lines going out from the launching region, that is, the black hole corona, are radial (e.g., Gracia et al. 2009). Moreover synthetic synchrotron maps built from such magnetohydrodynamic (MHD) models can reproduce the opening angle of M 87 up to a large distance from the black hole, and the change between center-brightening near the core to limb-brightening further away.

The standard accretion-jet model includes an advectiondominated accretion flow (ADAF) which takes place at least in the inner part of the accretion disk in AGNs, except for very high accretion efficiency (Narayan \& Yi 1994). The presence of this ADAF structure in the disk center does not prevent the launching of the internal jet as can be seen from observations near the black hole for radiogalaxies (see the Faraday rotation measures from observations of M 87 in Feng et al. 2016). In the high-accretion regime for AGNs, where a standard thin disk is found and powerful outflows are observed, both an internal jet and an outer disk wind coexist.

Numerical simulations in the framework of general relativity have been performed to model the inner jet formation assuming a large-scale magnetic field right from the beginning, mostly using monopolar configuration anchored in the ergosphere. Komissarov (2007) was able to ensure the viability of the Blandford-Znajek mechanism (Blandford \& Znajek 1977) and the possibility of building a relativistic particle outflow. Similar relativistic simulations of jets were obtained by McKinney \& Blandford (2009) where the central Poynting-fluxdominated force-free jet is self-consistently feeding the funnel of the accretion-disk wind created along the axis by the very high toroidal field in a magnetically arrested disk (MAD). McKinney et al. (2012) and Tchekhovskoy et al. (2011) have also derived a scaling law between the magnetic flux of the field threading the black hole and the mass-accretion rate on the horizon. This has been confirmed observationally by Zamaninasab et al. (2014). In addition, the jet power, directly related to the magnetic flux on the horizon, was calculated in their simulations, allowing for the derivation of a net flow efficiency between the accretion and the jet.

Beyond the launching region, the internal structure of magnetized and relativistic jets can be probed by relativistic MHD (RMHD) simulations, as has been done by Martí et al. (2016). They characterized the internal jet structure of overpressured, steady jets in connection with the dominant energy (internal, rest-mass, and magnetic). They showed that transverse equilibrium with a significant toroidal magnetic component implies a structure with a central spine and a surrounding layer with lower thermal and total pressures. Thus, we have strong clues, both from observations and simulations, that relativistic jets have a transverse structure. On the other hand, the jet launching mechanism associated with magnetic flux threading the black hole seems to produce only very light spine jets with rarified gas such that the inner outflow is force-free. The inner beam would be so light that it would be invisible at large distances. An alternative issue, in order to model the spine jet, is to extend the self-similar solutions for jet outflows in young stellar objects to general relativity. This model can be derived analytically and used as initial conditions in numerical simulations.

A self-similar model of the spine jet has been proposed by Meliani et al. (2006) for nonrotating black holes in Schwarzschild metric, extending the previous Newtonian self-similar model of Sauty \& Tsinganos (1994). The Meliani et al. (2010) model aimed to explain the dichotomy between FRI and FRII sources in terms of magnetic collimation efficiency. Then, Globus et al. (2014), partially generalized this model to a Kerr metric. However they fail to give an exact generalization of the magnetic collimation criterion linked to the efficiency of the magnetic rotator. In both cases, the authors assume that the light cylinder is sufficiently far from the spine jet to have its effects neglected. Physically, this means that 
both models cannot produce solutions consistent across the light cylinder.

In the present paper, we present a new extension of the nonrelativistic meridional self-similar solutions of Sauty \& Tsinganos (1994), both in Schwarzschild and Kerr metrics. This model can produce solutions for relativistic jets emerging from a spherical corona surrounding the central part of a Kerr black hole and its inner accretion disk.

Unlike previous models in a Schwarzschild or a Kerr metric, this new model for a nonrotating, as well as for a rotating black hole includes a self-consistent crossing of the light cylinder in the solution. The Alfvén Mach surfaces are not spherically symmetric. However, the Alfvén transition surface is spherical and includes the light cylinder.

In Sect. 2 we summarize the ideal MHD equations in the $3+1$ formalism and in Sect. 3 construct the model and derive its equations. In Sect. 4 we describe how to solve the equations and illustrate this procedure with various new solutions in a Schwarzschild metric. Then, four different solutions in a Kerr metric are presented in Sect. 5 with high Lorentz factor and different geometries potentially applicable to AGNs and $\gamma$-ray bursts (GRB). Some characteristics of those solutions of the model are discussed as well as how the magnetic collimation evolves with the rotation of the black hole.

\section{Steady axisymmetric relativistic MHD outflows}

\subsection{Kerr metric}

The first step in building self-similar solutions in relativistic flows is to define the metric. In fact the central massive black hole dominates the gravitational field in the near regions and determines completely the metric field. Thus, in Kerr metric, the geodesics are defined as follows.

$$
\begin{aligned}
\mathrm{d} s^{2}= & -\left(1-\frac{r_{s} r}{\rho^{2}}\right) c^{2} \mathrm{~d} t^{2}-\frac{2 r_{s} r c a}{\rho^{2}} \sin ^{2} \theta \mathrm{d} t \mathrm{~d} \phi \\
& +\frac{\rho^{2}}{\Delta} \mathrm{d} r^{2}+\rho^{2} \mathrm{~d} \theta^{2}+\frac{\Sigma^{2}}{\rho^{2}} \sin ^{2} \theta \mathrm{d} \phi^{2} .
\end{aligned}
$$

We have the usual notations of the elements,

$\Delta=r^{2}+a^{2}-r_{s} r$

$\rho^{2}=r^{2}+a^{2} \cos ^{2} \theta$

$\Sigma^{2}=\left(r^{2}+a^{2}\right)^{2}-a^{2} \Delta \sin ^{2} \theta$,

where $a=\frac{\mathcal{J}}{\mathcal{M} c}$ and $r_{s}=\frac{2 \mathcal{G M}}{c^{2}}$.

We note that $\mathcal{J}$ is the angular momentum of the massive central object, $\mathcal{M}$ is its mass, $h$ is the lapse function, $\omega$ is the angular velocity of zero angular momentum observers (ZAMO) and we use $a$ for the length-scale related to the angular momentum of the black hole (Kerr scale). We can define the dimensionless spin of the black hole $a_{H}$ in units of the gravitational radius $r_{s} / 2$ such that, $a_{H}=2 a / r_{s}$. Furthermore, $\boldsymbol{\beta}$ is the shift vector.

The lapse function $h$, the angular velocity $\omega$ of zero angular momentum observers (ZAMO) and the shift vector coordinates can be written as:

$h=\left(1-\frac{r_{s} r}{\rho^{2}}+\boldsymbol{\beta}^{\phi} \boldsymbol{\beta}_{\phi}\right)^{1 / 2}=\frac{\rho}{\Sigma} \sqrt{\Delta}$,

$\omega=\frac{a c r_{s} r}{\Sigma^{2}}, \quad \beta_{\phi}=-\frac{\omega}{c} \varpi^{2}, \quad \beta^{\phi}=-\frac{\omega}{c}$, with $\varpi=\frac{\Sigma}{\rho} \sin \theta$. The corresponding line elements for the Kerr metric are given in Appendix A.

\subsection{Maxwell's equations}

The next step is to define the electromagnetic field in this metric. Using covariant derivatives, we can write Maxwell's equations in Kerr space, assuming stationarity and axisymmetry,

$\nabla \cdot \boldsymbol{E}=4 \pi \rho_{e}$,

$\nabla \cdot \boldsymbol{B}=0$,

$\nabla \times(h \boldsymbol{E})=\left(\boldsymbol{B} \cdot \nabla \frac{\omega}{c}\right) \varpi \boldsymbol{\epsilon}_{\phi}$,

$\nabla \times(h \boldsymbol{B})=\frac{4 \pi h}{c} \boldsymbol{J}-\left(\boldsymbol{E} \cdot \nabla \frac{\omega}{c}\right) \varpi \boldsymbol{\epsilon}_{\phi}$,

where $\left(\boldsymbol{\epsilon}_{i}\right)_{i=1 \ldots 3}$ is the space orthonormal basis. We note that all quantities in the above equations are given in the ZAMO frame. We can split all vector fields in a poloidal component in the meridional plane and a toroidal one along the azimuthal direction. The poloidal magnetic field $\boldsymbol{B}_{\mathrm{p}}$ can be expressed in terms of the magnetic flux function $A$,

$\boldsymbol{B}_{\mathrm{p}}=\nabla \times\left(\frac{A}{\varpi} \boldsymbol{\epsilon}_{\phi}\right)$,

and using Faraday's law, we get the electric field,

$\nabla \times\left(h \boldsymbol{E}-\frac{\omega}{c} \nabla A\right)=0$.

We can introduce the electric potential, $\Phi$, but the electric field $\boldsymbol{E}$ is not directly proportional to the gradient of the electric potential,

$h \boldsymbol{E}=\frac{\omega}{c} \nabla A-\nabla \Phi$.

The condition of ideal MHD for infinite electrical conductivity leads to

$\boldsymbol{E}+\frac{\boldsymbol{V} \times \boldsymbol{B}}{c}=0$.

We note that $\nabla$ is the covariant derivative on a space hypersurface; see Appendix B for the expression of its coordinates.

\subsection{Equations of motion}

In the Kerr metric, the $3+1$ formalism gives the equation for mass conservation, the Euler equation, and the energy conservation, respectively,

$\nabla \cdot\left(\rho_{0} \gamma h \boldsymbol{V}\right)=0$

$\rho_{0} \gamma(\boldsymbol{V} \cdot \nabla)(\gamma \xi \boldsymbol{V})+\rho_{0} \xi \gamma^{2}\left[c^{2} \nabla \ln h+\frac{\varpi \omega V^{\hat{\phi}}}{h} \nabla \ln \omega\right]$

$$
+\nabla P=\rho_{e} \boldsymbol{E}+\frac{\boldsymbol{J} \times \boldsymbol{B}}{c},
$$

$\gamma^{2} \rho_{0} \xi c\left[\boldsymbol{V} \cdot \nabla \ln (\gamma \xi h)+\frac{\omega \varpi V^{\hat{\phi}}}{h c^{2}} \boldsymbol{V} \cdot \nabla \ln \omega\right]=\frac{\boldsymbol{J} \cdot \boldsymbol{E}}{c}$.

Here $V^{\hat{\phi}}$ is the toroidal component of the bulk flow speed as seen by the ZAMO. The factor $\gamma$ is the bulk Lorentz factor, $\rho_{0}$ is 
the mass density, and $\xi c^{2}$ the specific enthalpy measured in the comoving frame of the outflow, that contains kinetic enthalpy of perfect relativistic gas $\xi_{K}$ and some heating term $Q / c^{2}$.

$\xi=\xi_{K}+\frac{Q}{c^{2}}$

For the kinetic enthalpy we use the Taub-Matthews approximation of ideal fluid equation of state; for more details see Taub (1948), Meliani et al. (2004) and Mignone et al. (2005).

$\xi_{K}=\frac{5}{2}\left(\frac{P}{\rho_{0} c^{2}}\right)+\sqrt{1+\left(\frac{3 P}{2 \rho_{0} c^{2}}\right)^{2}}$.

The energy conservation has been derived in the frame of the ZAMO and equivalently the first law of thermodynamics can be obtained by projecting the conservation of the energymomentum tensor along the fluid 4-velocity but in the comoving frame. Assuming infinite conductivity, the contribution of the electromagnetic field is null and only the thermal energy affects the variation of the enthalpy of the fluid, giving,

$\rho_{0}\left(\boldsymbol{V}_{\mathrm{p}} \cdot \nabla\right)\left(\xi c^{2}\right)=\left(\boldsymbol{V}_{\mathrm{p}} \cdot \nabla\right) P$.

\subsection{Constants of motion}

Under the assumptions of steadiness and axisymmetry, the MHD equations in general relativity can be partially integrated to yield several field/streamline constants (Beskin 2010). We already deduced those constants including the magnetic field with the same formalism in Cayatte et al. (2014). Here we present the derivation of the equations, following the notations of Tsinganos (1982), in order to compare with previous self-similar models, for example, Meliani et al. (2006) and give the choice of the first integrals.

Steady and axisymmetric flows are characterized by a function $A$ that defines the geometry of the magnetic flux surfaces. In the poloidal plane, field lines are lines of constant magnetic flux $A$ and first integrals will be functions of $A$, among which the mass flux $\Psi$. The poloidal velocity can be expressed in terms of $\Psi$,

$4 \pi \rho_{0} \gamma h \boldsymbol{V}_{\mathrm{p}}=\nabla \times\left(\frac{\Psi}{\varpi} \boldsymbol{\epsilon}_{\phi}\right)$.

The frozen-in condition for ideal MHD flows, gives, in the toroidal direction, combined with Eq. (11),

$4 \pi \rho_{0} \gamma h \boldsymbol{V}_{\mathrm{p}}=\Psi_{A} \boldsymbol{B}_{\mathrm{p}}$,

where $\Psi_{A} \equiv \mathrm{d} \Psi / \mathrm{d} A$ is the magnetic to mass flux ratio.

The poloidal components of the law of flux freezing (Eq. (14)) give in turn the iso-rotation law,

$\Omega-\omega=\frac{h V^{\hat{\phi}}}{\varpi}-\frac{\Psi_{A} B^{\hat{\phi}}}{4 \pi \rho_{0} \gamma \varpi}$,

where $\Omega(A) \equiv c \mathrm{~d} \Phi / \mathrm{d} A$, which is the isorotation frequency, constant along each magnetic flux tube.

By integrating the Euler equation in the toroidal direction, we get the conservation of the angular momentum flux $L(A)$,

$L=\varpi\left(\gamma \xi V^{\hat{\phi}}-\frac{h B^{\hat{\phi}}}{\Psi_{A}}\right)$
The last equation to integrate is the energy conservation. In other words, we may take the Euler equation projected along the time axis of the $3+1$ decomposition, and integrate it under the hypothesis of steadiness,

$\mathcal{E}-L \omega=\gamma \xi h c^{2}-\frac{h \varpi(\Omega-\omega)}{\Psi_{A}} B^{\hat{\phi}}$.

\subsection{Toroidal fields}

Using the three last integrals of motion, we may express the toroidal components of the velocity and the magnetic fields and the enthalpy density as functions of these first integrals and the poloidal components. Using the standard procedure of inversion we get

$\varpi \frac{h B^{\hat{\phi}}}{\Psi_{A}}=\frac{L\left[h^{2} c^{2}+\varpi^{2} \omega(\Omega-\omega)\right]-\varepsilon \varpi^{2}(\Omega-\omega)}{\left(M_{\mathrm{Alf}}^{2}-h^{2}\right) c^{2}+\varpi^{2}(\Omega-\omega)^{2}}$,

$\varpi \gamma \xi V^{\hat{\phi}}=\frac{M_{\mathrm{Alf}}^{2} L c^{2}-(\mathcal{E}-L \Omega) \varpi^{2}(\Omega-\omega)}{\left(M_{\mathrm{Alf}}^{2}-h^{2}\right) c^{2}+\varpi^{2}(\Omega-\omega)^{2}}$,

$\gamma h \xi=\frac{M_{\mathrm{Alf}}^{2}(\mathcal{E}-L \omega)-h^{2}(\mathcal{E}-L \Omega)}{\left(M_{\mathrm{Alf}}^{2}-h^{2}\right) c^{2}+\varpi^{2}(\Omega-\omega)^{2}}$,

where we have defined the poloidal Alfvén Mach number,

$M_{\text {Alf }}^{2}=h^{2} \frac{V_{\mathrm{p}}^{2}}{V_{\mathrm{Alf}}^{2}}=\frac{4 \pi h^{2} \rho_{0} \xi \gamma^{2} V_{\mathrm{p}}^{2}}{B_{\mathrm{p}}{ }^{2}}=\frac{\xi \Psi_{A}^{2}}{4 \pi \rho_{0}}$.

This definition of the poloidal Alfvén Mach number is consistent with the definition used by Meliani et al. (2006) and includes the lapse function. This is also the definition taken by Breitmoser \& Camenzind (2000) because the velocity, $h V_{\mathrm{p}}$, calculated with the universal time is continuous across the event horizon.

The numerator and denominator of Eq. (25) are zero at the Alfvén transition surface, if the following two equations are satisfied,

$\left.M_{\mathrm{Alf}}^{2}\right|_{\mathrm{a}}=h_{\mathrm{a}}^{2}\left[1-\frac{\varpi_{\mathrm{a}}^{2}\left(\Omega-\omega_{\mathrm{a}}\right)^{2}}{h_{\mathrm{a}}^{2} c^{2}}\right]$,

$\frac{\varpi_{\mathrm{a}}^{2}\left(\Omega-\omega_{\mathrm{a}}\right)^{2}}{h_{\mathrm{a}}^{2} c^{2}}=\frac{L\left(\Omega-\omega_{\mathrm{a}}\right)}{\left(\mathcal{E}-L \omega_{\mathrm{a}}\right)}$.

The denominators of Eqs. (26) and (27) are identical to the one of Eq. (25) and there numerators are a linear combination of Eqs. (29) and (30). Hence, the numerators and the denominators of Eqs. (26) and (27) are also zero at the Alfvén transition surface.

We can reformulate the above equations by changing variables. We rescale the cylindrical radius with $\operatorname{ch} /(\Omega-\omega)$ leading to the dimensionless cylindrical radius $x$ and introduce the parameter $x_{\mathrm{MR}}$,

$x=\frac{\varpi(\Omega-\omega)}{h c}, x_{\mathrm{MR}}^{2}=\frac{L(\Omega-\omega)}{(\mathcal{E}-L \omega)}$.

Hence we can write,

$B^{\hat{\phi}}=\frac{-(\mathcal{E}-L \omega) \Psi_{A}}{c x} \frac{x^{2}-x_{\mathrm{MR}}^{2}}{M_{\mathrm{Alf}}^{2}-h^{2}\left(1-x^{2}\right)}$, 
$h \gamma \xi \frac{V^{\hat{\phi}}}{c}=\frac{(\mathcal{E}-L \omega)}{c^{2} x} \frac{M_{\mathrm{Alf}}^{2} x_{\mathrm{MR}}^{2}-\left(1-x_{\mathrm{MR}}^{2}\right) h^{2} x^{2}}{M_{\mathrm{Alf}}^{2}-h^{2}\left(1-x^{2}\right)}$,

$\gamma h \xi=\frac{(\mathcal{E}-L \omega)}{c^{2}} \frac{M_{\mathrm{Alf}}^{2}-h^{2}\left(1-x_{\mathrm{MR}}^{2}\right)}{M_{\mathrm{Alf}}^{2}-h^{2}\left(1-x^{2}\right)}$.

The second condition, Eq. (30), at the Alfvén transition surface becomes, keeping the first one unchanged,

$\left.x^{2}\right|_{\mathrm{a}}=\left.x_{\mathrm{MR}}^{2}\right|_{\mathrm{a}}$.

In Kerr metric, the parameter $x_{\mathrm{MR}}^{2}$ is an extension of $x_{\mathrm{A}}^{2}$ defined by Meliani et al. (2006). It measures the amount of energy carried by the electromagnetic field. This is the energy flux of the magnetic rotator (MR) divided by the total energy flux of the outflow in the co-rotating frame, $\mathcal{E}-L \omega$. This new parameter $x_{\mathrm{MR}}$, conversely to the previous $x_{\mathrm{A}}$, is not any more constant along a field line, since $\omega$ is not an integral of the motion.

\section{Model equations}

\subsection{Angular expansion}

The MHD equations and the metric constitute a coupled set of highly nonlinear equations that cannot be solved analytically. The approach followed so far for Newtonian flows has been to look for solutions with separable variables in the frame of self-similarity. However, this technique cannot be applied in the frame of general relativity due to the complexity of the metric even for the simpler cases of a Schwarzschild or a Kerr metric. Instead, we may model the jet close to its symmetry axis, that is, to describe the spine jet, by expanding all variables with $\sin \theta$ to second order.

Along the polar axis where $\varpi$ and $\theta$ go to zero, we may define the spherical Alfvén radius to be the distance $r_{\star}$ from the center where the Alfvén transition surface condition, $M_{\text {Alf }, \theta=0}^{2}=h_{\star}^{2}$, applies. The subscript $\star$ denotes the value of a physical quantity at the Alfvén transition surface, along the polar axis. We shall use this location to write all our quantities in dimensionless form. Thus, the dimensionless spherical radius is

$R=\frac{r}{r_{\star}}$

At $R=1$, the velocity is $V_{\star}$, the magnetic field $B_{\star}$, the density $\rho_{\star}$, the enthalpy $\xi_{\star}$ and the lapse function $h_{\star}$. Because of the Alfvén transition along the polar axis, we have,

$B_{\star}^{2}=4 \pi \gamma_{\star}^{2} \rho_{\star} \xi_{\star} V_{\star}^{2}$.

Thus the dimensionless magnetic flux function $\alpha$ is defined as,

$\alpha=\frac{2}{r_{\star}^{2} B_{\star}} A$.

Moreover we can expand to the second order the metric of the system in dimensionless form using the characteristic dimensions of the system defined in the previous Section. This introduces the two following new parameters,

$\mu=\frac{r_{s}}{r_{\star}}, \quad l=\frac{a}{r_{\star}}=\frac{\mathcal{J}}{\mathcal{M c r} r_{\star}} \Rightarrow \frac{2 a}{r_{\star}}=\frac{2 l}{\mu}$,

which are respectively the Schwarzschild radius in units of the Alfvén radius and the dimensionless black hole spin.
Another dimensionless parameter is needed to describe the gravitational potential, as in the classical model. This parameter $v$ represents the escape speed at the Alfvén point along the polar axis in units of $V_{\star}$. Then, the value of $V_{\star}$ is fixed by the following condition,

$v=\frac{V_{\mathrm{esc}, \star}}{V_{\star}}=\sqrt{\frac{2 \mathcal{G M}}{r_{\star} V_{\star}^{2}}} \Rightarrow V_{\star}^{2}=\frac{\mu}{v^{2}} c^{2}$.

Thus, to second order in $\sin \theta$ the ZAMO angular velocity and the lapse function are written as,

$$
\begin{aligned}
& \omega=\frac{l c \mu R}{r_{\star}\left(R^{2}+l^{2}\right)^{2}}\left(1+\frac{l^{2} h_{z}{ }^{2}}{R^{2}+l^{2}} \sin ^{2} \theta\right), \\
& h=\sqrt{1-\frac{\mu R}{R^{2}+l^{2}}}\left(1-\frac{\mu l^{2} R}{2\left(R^{2}+l^{2}\right)^{2}} \sin ^{2} \theta\right) .
\end{aligned}
$$

In order to simplify our notation, we define the lapse function along the polar axis,

$h_{z}(R)=h(R, \theta=0)=\sqrt{1-\frac{\mu R}{R^{2}+l^{2}}}$,

and the polar shift of the metric,

$\omega_{z}(R)=\omega(R, \theta=0)=\frac{l c \mu R}{r_{\star}\left(R^{2}+l^{2}\right)^{2}}$.

See Appendix A for details.

It will be useful to introduce the dimensionless polar shift function (see also Eq. (59)),

$\bar{\omega}_{z}(R)=\frac{\omega_{z} r_{\star}}{V_{\star} h_{\star}}=\frac{l \sqrt{\mu} v R}{h_{\star}\left(R^{2}+l^{2}\right)^{2}}$.

We also expand the magnetic flux function to second order in $\sin \theta$. The magnetic flux is an even function which is zero along the polar axis due to axisymmetry and because of the symmetry around the equatorial plane. Thus all odd orders are zero and the first nonvanishing even order is the second order in colatitude. If we keep the lowest order in the expansion we get

$\alpha(R, \theta)=f(R) \sin ^{2} \theta$,

where $f$ is the inverse of the classical expansion factor for solar coronal holes (see Tsinganos \& Sauty 1992). This expansion, similarly to the classical self similar model of Sauty \& Tsinganos (1994), is equivalent to a hypothesis of separation of the variables in the magnetic flux function.

Thus from Eq. (A.17), the cylindrical radius can also be seen as an expansion in the magnetic flux. This is physically more meaningful as the magnetic flux is constant on a given mass flux tube. Moreover, several free integrals depend solely on this magnetic flux. We define the dimensionless cylindrical radius $G$ in units of the polar Alfvén radius as,

$G(R)=\sqrt{\frac{R^{2}+l^{2}}{f(R)}}$. forms,

The cylindrical radius can be written in the various following

$\varpi^{2}=r_{\star}^{2}\left(R^{2}+l^{2}\right) \sin ^{2} \theta=r_{\star}^{2} G^{2} \alpha=G^{2} \varpi_{a}^{2}$. 
We can also write the metric as an expansion in $\alpha$ (see also Appendix A),

$$
\begin{aligned}
& \omega=\frac{l c \mu R}{r_{\star}\left(R^{2}+l^{2}\right)^{2}}\left(1+\frac{l^{2} h_{z}^{2} G^{2}}{\left(R^{2}+l^{2}\right)^{2}} \alpha\right), \\
& h=\sqrt{1-\frac{\mu R}{R^{2}+l^{2}}}\left(1-\frac{\mu l^{2} R G^{2}}{2\left(R^{2}+l^{2}\right)^{3}} \alpha\right) .
\end{aligned}
$$

Of course we can always reverse our point of view and go back to the expansion in $\theta$. This would be the case if we wanted to use the steady analytical solution as initial conditions for numerical simulations.

We can parametrize the geometry of the flux tubes with the logarithm derivative of $f$ denoted $F$,

$F=\frac{\mathrm{d} \ln f}{\mathrm{~d} \ln R}=2\left(\frac{R^{2}}{R^{2}+l^{2}}-\frac{\mathrm{d} \ln G}{\mathrm{~d} \ln R}\right)$.

The angle $\chi$ of the magnetic poloidal field line with the radial direction (see Sauty et al. 1999) is given in our metric by,

$\tan \chi=\frac{\sqrt{R^{2}+l^{2}-\mu R}}{2 R} F \tan \theta$.

\subsection{Choice of the Alfvén surface and pressure}

We can expand all physical quantities to the first order in $\alpha$. Thus the Alfvén number is given by,

$M_{\mathrm{Alf}}=M(R)\left(1+M_{1}(R) \alpha\right)$.

Contrary to previous self-similar models, the Alfvén number cannot be spherically symmetric because of the presence of the cylindrical radius in units of the "light cylinder" $x$, in the numerator and the denominator of Eqs. (32)-(34). This is induced by the regularity conditions, Eqs. (29) and (30), and the sphericity of the Alfvén surface. The surface $x=1$ is the so-called outer "light cylinder". Of course this surface may not be exactly cylindrical if $x$ depends also on $\alpha$, which may be the case for instance close to the black hole where $\omega$ has a strong dependence on $\alpha$ or if $\Omega$ is not constant with $\alpha$. Therefore, this is rather a light surface, but for the sake of simplicity we refer to it as a "light cylinder" in the rest of the text.

Similarly, the pressure can be expanded to first order,

$P(R, \alpha)=P_{0}+\frac{\gamma_{\star}^{2} \rho_{0 \star} \xi_{\star} V_{\star}^{2}}{2} \Pi(R)(1+K(R) \alpha)$,

where $P_{0}$ is a constant.

In order to simplify and as a first step, we assume for both equations that the radial dependence of the nonpolar component of the Alfvén number and the pressure are simply constant, $M_{1}(R)=m_{1}=\mathrm{cst}, K(R)=\kappa=\mathrm{cst}$. Thus,

$M_{\mathrm{Alf}}=M(R)\left(1+m_{1} \alpha\right)$.

We note that we have $m_{1}=0$ in previous models; see Meliani et al. (2006) and Globus et al. (2014).

\subsection{Choice of the free integrals}

Free integrals are also expanded to the first order in the magnetic flux. The mass to magnetic flux ratio is similar to the one in the classical case, expanded as,

$\Psi_{A}^{2}(\alpha)=\frac{4 \pi \rho_{0 \star} h_{\star}^{2}}{\xi_{\star}}(1+\delta \alpha)$

where $\delta$ is a free parameter describing the deviations from spherical symmetry of the ratio number density/enthalpy as in Meliani et al. (2006) and not of the density itself, conversely to Sauty \& Tsinganos (1994).

The total angular momentum loss flux density is given by

$\boldsymbol{J}=\gamma \rho_{0} L h \boldsymbol{V}_{\mathrm{p}}=\frac{L \Psi_{A}}{4 \pi} \boldsymbol{B}_{\mathrm{p}}$.

Thus it is natural to expand the quantity $L \Psi_{A}$ rather than $L$ itself. $L \Psi_{A}$ is also the poloidal current density along the polar axis and writes as

$L \Psi_{A}=\lambda h_{\star} B_{\star} r_{\star} \alpha$.

The isorotation law can be expanded to first order as well as the total energy,

$\Omega=\Omega_{\star}\left(1+w_{1} \alpha\right)$,

and

$\mathcal{E}=\mathcal{E}_{\star}\left(1+e_{1} \alpha\right)$,

where we see from Eq. (24) that $\mathcal{E}_{\star}=h_{\star} \gamma_{\star} \xi_{\star} c^{2}$.

Although we have some freedom with the choice of $w_{1}$ and $e_{1}$, we could choose $e_{1}=0$ and $w_{1}=-\delta / 2$ to restrict ourselves to the values of the previous models, in particular in Schwarzschild metric; see Meliani et al. (2006) and Meliani et al. (2010). In fact, the isorotation function $\Omega$ does not need to be expanded beyond the zeroth-order term because $\Omega$ always appears multiplied by another quantity as in $(\Omega-\omega) \varpi$ or $L \Omega$. Thus, the value of $w_{1}$ is free and does not affect the solution. Conversely, the value of $e_{1}$ affects the whole dynamics and we shall study the effects of its variation in a future publication. We already discussed the fact that taking a weak dependence of $\Omega$ on $\alpha$ has the advantage of minimizing the variation of the "light cylinder" near the base of the jet. Thus for the sake of simplicity, we shall study here the case where $e_{1}=0$ and $w_{1}=0$.

\subsection{Constraints on the Alfvén Mach number, the isorotation law, and the angular momentum flux}

The value of $m_{1}$ is, in fact, determined by the prescription to cross the Alfvén transition surface. In order for the denominator in Eqs. (25)-(27) to vanish at the Alfvénic transition, the two following relations given in Eqs. (29) and (30) must be fulfilled. They can be expanded to first order. For the first regularity condition we get,

$M_{\mathrm{Alf}} \mathrm{l}_{\mathrm{a}}=h_{\star}\left(1+m_{1} \alpha\right)$ with $m_{1}=-\frac{\mu}{2}\left(\frac{\lambda^{2}}{v^{2}}+\frac{l^{2}}{\left(1+l^{2}\right)^{3}}\right)$.

The first term in the right part of Eq. (61) is due to the "light cylinder", and the second one to the nonsphericity of the gravitational field in Kerr metric. $m_{1}$ is negligible whenever the 
rotational speed $\lambda V_{\star}$ is sub-relativistic and either the $\mu$ parameter or the angular momentum of the black hole are negligible too. Since $m_{1}<0$, there is a limiting field line where we have $M_{\text {Alf }}=0$ since the magnetic flux increases going out from the polar axis.

To apply the second regularity condition we use the numerator of Eq. (25) and we get to the first order in $\alpha$ :

$\Omega_{\star}-\omega_{\star}=\frac{\lambda V_{\star} h_{\star}}{r_{\star}}$.

Thus we can write

$\varpi(\Omega-\omega)=G(R) \sqrt{\alpha} \lambda V_{\star} h_{\star} \Lambda(R)$,

where,

$\Lambda(R)=\left[1+\frac{\sqrt{\mu} v l}{\lambda h_{*}}\left(\frac{1}{\left(1+l^{2}\right)^{2}}-\frac{R}{\left(R^{2}+l^{2}\right)^{2}}\right)\right]$.

The regularity conditions on the Alfvén surface fixes the value of $m_{1}$. Thus the critical Alfvén surface is a sphere, as in previous meridional self-similar models. We warn, however, that the Alfvén transition surface is a generalized or modified Alfvén surface as it takes into account the modification by the "light cylinder".

Simultaneously, surfaces of constant Poloidal Alfvén Mach Number, $M_{\text {Alf }}=$ const. (see Eq. (55)) are not spherical surfaces, conversely to the one defined by Meliani et al. (2006). Two effects modify it; first the "light cylinder" effect, which was neglected in Meliani et al. (2006) and Globus et al. (2014), and second the frame-dragging effect (Lense-Thirring).

\subsection{Expansion of the velocity and magnetic fields}

The model is obtained using an expansion to the second order for $\sin \theta$ in the Euler equation. Due to axisymmetry, first-order terms are zero along $r$ and $\phi$ while the antisymmetry along $\theta$ gives the zeroth and second orders as null along the colatitude.

Then, for the poloidal velocity field, this gives

$$
\begin{aligned}
V^{\hat{r}}= & \frac{V_{\star} M^{2}}{h_{\star}^{2} G^{2}}\left\{1+\sin ^{2} \theta\left[\frac{1}{2}\left(\frac{l^{2} h_{z}^{2}}{R^{2}+l^{2}}-1\right)\right.\right. \\
& \left.\left.+\frac{R^{2}+l^{2}}{G^{2}}\left(\frac{\lambda^{2} \mu}{v^{2}}\left(\frac{\Lambda^{2} N_{B}}{D}+\frac{\bar{\omega}_{z}}{\lambda}\right)-e_{1}-\frac{\delta}{2}+2 m_{1}\right)\right]\right\} \\
V^{\hat{\theta}}= & -\frac{V_{\star} h_{z} M^{2} \sqrt{R^{2}+l^{2}} F}{2 h_{\star}^{2} R G^{2}} \sin \theta .
\end{aligned}
$$

And for the poloidal magnetic field, we get

$$
\begin{aligned}
& B^{\hat{r}}=\frac{B_{\star}}{G^{2}}\left[1+\frac{1}{2}\left(\frac{l^{2} h_{z}^{2}}{R^{2}+l^{2}}-1\right) \sin ^{2} \theta\right], \\
& B^{\hat{\theta}}=-\frac{B_{\star} h_{z} F \sqrt{R^{2}+l^{2}}}{2 G^{2} R} \sin \theta .
\end{aligned}
$$

Now from Eqs. (25) and (26), we can calculate to the first order in $\sin \theta$ the toroidal components of fields

$$
\begin{aligned}
V^{\hat{\phi}} & =-\frac{\lambda V_{\star} h_{z} \Lambda N_{V}}{h_{\star} G^{2} D} \sqrt{R^{2}+l^{2}} \sin \theta, \\
B^{\hat{\phi}} & =-\frac{\lambda B_{\star} h_{\star} \Lambda N_{B} \sqrt{R^{2}+l^{2}}}{h_{z} D G^{2}} \sin \theta,
\end{aligned}
$$

where the functions $N_{V}, N_{B}$, and $D$ have been generalized,

$$
\begin{aligned}
& N_{V}=\frac{M^{2}}{h_{\star}^{2} \Lambda}-G^{2}, \\
& N_{B}=\frac{h_{z}^{2}}{h_{\star}^{2} \Lambda}-G^{2}, \\
& D=\frac{h_{z}^{2}-M^{2}}{h_{\star}^{2}} .
\end{aligned}
$$

\subsection{Expansion of the enthalpy, densities, and electric field}

We used Eq. (27) to deduce the enthalpy,

$$
\gamma h \xi c^{2}=\gamma_{\star} h_{\star} \xi_{\star} c^{2}\left[1+\alpha\left(e_{1}-\frac{\lambda^{2} \mu}{v^{2}}\left(\frac{\Lambda^{2} N_{B}}{D}+\frac{\bar{\omega}_{z}}{\lambda}\right)\right)\right],
$$

and the mass density is given by

$$
\begin{aligned}
\gamma^{2} \rho_{0} \xi & =\gamma_{\star}^{2} \rho_{0 \star} \xi \star \frac{h_{\star}^{4}}{h_{z}^{2} M^{2}}\left[1+\sin \theta^{2}\left\{\frac{\mu l^{2} R}{\left(R^{2}+l^{2}\right)^{2}}\right.\right. \\
& \left.\left.+\frac{R^{2}+l^{2}}{G^{2}}\left(2 e_{1}-2 m_{1}+\delta-\frac{2 \lambda^{2} \mu}{v^{2}}\left(\frac{\Lambda^{2} N_{B}}{D}+\frac{\bar{\omega}_{z}}{\lambda}\right)\right)\right\}\right] .
\end{aligned}
$$

In GRMHD, we also need the expressions of the electric field and the charge density. The electric field is a second-order term for the radial component and a first-order term for the $\theta$-component,

$$
\begin{aligned}
E^{\hat{r}} & =-\frac{\lambda V_{\star} h_{\star} B_{\star}}{2 c} \frac{\left(R^{2}+l^{2}\right) F \Lambda}{R G^{2}} \sin ^{2} \theta, \\
E^{\hat{\theta}} & =-\frac{\lambda V_{\star} h_{\star} B_{\star}}{c} \frac{\Lambda \sqrt{R^{2}+l^{2}}}{h_{z} G^{2}} \sin \theta .
\end{aligned}
$$

Using Maxwell-Gauss Eq. (7), we calculate the charge density from the divergence of the above electric field, to zeroth order only,

$\rho_{\mathrm{e}}=-\frac{\lambda V_{\star} B_{\star} h_{\star}}{2 \pi r_{\star} c} \frac{\Lambda}{h_{z} G^{2}}$.

With all these quantities we are able to expand the Euler equation. The radial component is expanded to the second order and the colatitude component to the first order. From the expansion of poloidal components in the Euler equation (Eq. (16)) and using Eq. (51), we can reverse the system to get the equations of the model (see Appendix C for details).

\section{7. "Light cylinder"}

The rescaling value $c h /(\Omega-\omega)$ for the cylindrical radius used in Eqs. (32)-(34) has been defined by Meliani et al. (2006) as the "light cylinder". It is a surface of revolution $\Sigma_{\mathrm{LC}}$ where,

$x^{2}=\frac{\varpi^{2}(\Omega-\omega)^{2}}{h^{2} c^{2}}=1$.

On the "light cylinder", the electric field $|\mathbf{E}|$ is equal to the poloidal component of the magnetic field $\left|\mathbf{B}_{\mathrm{p}}\right|$. In the present publication, $\Sigma_{\mathrm{LC}}$ designed the external "light cylinder", that is, $x=+1$, though, strictly speaking, this is not a cylinder as explained earlier, but a surface of revolution. This external "light cylinder" is outside the Alfvén surface since the denominator of 
Eqs. (32)-(34) is equal to $M_{A l f}^{2}$ on the "light cylinder", is negative before crossing the Alfvén transition surface, and is positive after crossing it. At large distance in the jet, the lapse function $h$ goes to unity and $\Omega-\omega$ tends to $\Omega$, which is assumed constant in our model. Thus, $\Sigma_{\mathrm{LC}}$ is located on a constant cylindrical radius along the $z$ axis, becoming a real cylinder.

From the iso-rotation law, we get,

$\frac{V^{\hat{\phi}}}{c}=x+\frac{\Psi_{A} B^{\hat{\phi}}}{4 \pi \rho_{0} \gamma h c}=x+\frac{V_{\mathrm{p}}}{c} \frac{B^{\hat{\phi}}}{B_{\mathrm{p}}}$.

As in special relativity, the second term of Eq. (79) cannot be neglected in the vicinity of the "light cylinder". The sign of $B^{\hat{\phi}}$ is such that $V^{\hat{\phi}}$ always remains less than the speed of light (Vlahakis 2015). Moreover after crossing the "light cylinder" one of the two following conditions must be fulfilled. Either, we have $\left|B^{\hat{\phi}}\right| » B_{\mathrm{p}}$ or $V_{\mathrm{p}} \gg V^{\hat{\phi}}$, or both.

The term $x$ was neglected in the equation of the previous relativistic meridional-self-similar models, Meliani et al. (2006); Globus et al. (2014). Hence, these models could not produce jets crossing the "light cylinder". Conversely, in this model this quantity is taken into account. We assume an expansion in $\sin (\theta)$ of this quantity.

Contrary to the two previous models we can choose the dependence of the isorotation frequency with the magnetic flux (see discussion on Eq. (59)) and this choice will not affect the solution. Thus, if $\Omega$ does not depend strongly on the magnetic flux $A$, even at the base of the jet, the ratio $h /(\Omega-\omega)$ will be nearly constant. The reason for this is that $\varpi$ is larger than the Alfvén radius, which is at least a few times the Schwarzschild radius. As a consequence, the departure of $\Sigma_{\mathrm{LC}}$ from a real cylinder is unnoticeable.

\subsection{Domain of validity}

The equations of the model are the result of an inversion of the expanded conservation equations. Therefore, it will be useful to quantify the relative error of the expansion we made in order to properly analyze our results and to obtain the domain of validity of these results. To have an idea of the domain of validity, we quantify the rest in the expansion of the momentum equation, for each force $\mathbf{F}^{i}(R, \sin \theta)$,

$$
\begin{aligned}
\mathbf{F}^{i}(R, \sin \theta)= & \mathbf{F}_{0}^{i}(R)+\mathbf{F}_{1}^{i}(R) \sin \theta+\mathbf{F}_{2}^{i}(R) \sin ^{2} \theta \\
& +\mathbf{R}^{i}(R, \sin \theta) \sin ^{3} \theta,
\end{aligned}
$$

where $\mathbf{F}$ is one of the following forces: gravitational, centrifugal, inertial, electric or magnetic pressure, and so on. We define a new function in order to map the relative error:

$\mathbf{R}^{i}(R, \sin \theta) \underset{\theta \rightarrow 0}{\sim} \mathbf{g}^{i}(R)$.

For example, in the case of the electric force, in Schwarzschild metric, we get, assuming solid rotation $\left(w_{1}=0\right)$,

$$
\begin{aligned}
\left|\mathbf{R}_{\mathrm{El}}(R, \sin \theta)\right|= & \frac{B_{\star}^{2}}{4 \pi r_{\star}} \frac{\lambda^{2} h_{\star}^{2} \mu}{v^{2}} \frac{R}{h_{z}^{2} G^{4}}\left(\frac{F^{2} h_{z}^{2}}{2}+\frac{F h_{z}^{2}}{2}-3+\frac{\mathrm{d} F}{\mathrm{~d} R}\right) \\
& \times \sqrt{1+\sin ^{2} \theta\left(\frac{F^{2} h_{z}^{2}}{4}-1\right)}
\end{aligned}
$$

The relative error on the electric force which tends to zero in the asymptotic regime of cylindrical jets is defined as

err $=\frac{\left|\mathbf{R}^{\mathrm{El}}(R, \sin \theta) \sin ^{3} \theta\right|}{\left|\mathbf{F}^{\mathrm{El}}(R, \sin \theta)\right|}$.

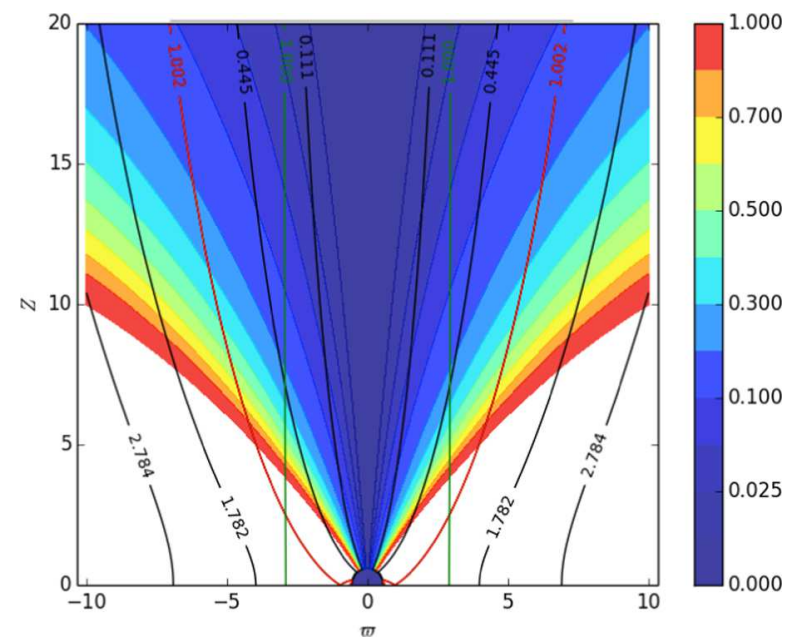

Fig. 1. Relative error on the electric force for a recollimating oscillating solution in Kerr metric (K1, see Sect. 5.1). Color isocontours correspond to the relative error in the electric force. Field lines anchored into the black hole magnetosphere and in the accretion disk are plotted in black solid lines. The limiting field line between the inner jet coming from black hole corona and the outflow outgoing from the accretion disk is plotted in red. The "light cylinder" is indicated by a green solid line. The cylindrical radius and the distance above the equatorial plane are in units of Schwarzschild radius.

Even at the base of the jet this error can be reduced, as can be seen in Fig. 1 for the solution in Kerr metric presented in Sect. 5.1 when the co-latitude is less than $30^{\circ}$. To get an estimate of the error in the expanded forces, we should add all relative error terms or take the largest one. This gives an estimate of the domain of validity of the solutions for a given set of parameters. We postpone the full error analysis for a future paper.

\subsection{The magnetic collimation efficiency, $\epsilon$}

By writing the first law of thermodynamics in the frame of the fluid along streamlines of an axisymmetric flow, we can construct a constant of the motion, as in the classical case. The first law of thermodynamics reduces to the adiabatic law if the heating is included in some effective enthalpy (see Eq. (20)). Thus $\left(\xi c^{2}\right)$ is an effective specific enthalpy, like for polytropic flows where the enthalpy also hides the heating (cf. Sauty \& Tsinganos 1994) but generalized for relativistic outflows (see Eq. (18)). Using Eq. (28), we can rewrite the first law of thermodynamics in the following form,

$\left.\xi \Psi_{A}^{2} c^{2} \frac{\mathrm{d} \xi}{\mathrm{d} R}\right|_{\alpha=\mathrm{cst}}=\left.4 \pi M_{A l f}^{2} \frac{\mathrm{d} P}{\mathrm{~d} R}\right|_{\alpha=\mathrm{cst}}$.

As the magnetic-to-mass-flux ratio and the total energy flux are constant along each streamline, this is equivalent to

$$
\left.\frac{\mathrm{d}\left(\Psi_{A}^{2} \xi^{2} c^{2}\right)}{\mathrm{d} R}\right|_{\alpha=\mathrm{cst}}=\left.8 \pi M_{A l f}^{2} \frac{\mathrm{d} P}{\mathrm{~d} R}\right|_{\alpha=\mathrm{cst}} .
$$

We note that $\Psi_{A} \xi c^{2}$ is proportional to the thermal energy. If we write

$\Psi_{A}^{2} \xi^{2} c^{2}=\left[\Psi_{A}^{2} \xi^{2} c^{2}\right]_{0}(R)+\alpha\left[\Psi_{A}^{2} \xi^{2} c^{2}\right]_{1}(R)$,

and using the expressions of the pressure and the Mach number, we get an equation of the form

$\frac{\mathrm{d}\left[\Psi_{A}^{2} \xi^{2} c^{2}\right]_{0}}{\mathrm{~d} R}+\alpha \frac{\mathrm{d}\left[\Psi_{A}^{2} \xi^{2} c^{2}\right]_{1}}{\mathrm{~d} R}=B_{\star}^{2} M^{2} \frac{\mathrm{d} \Pi}{\mathrm{d} R}\left[1+\left(\kappa+2 m_{1}\right) \alpha\right]$. 
We see, as in the classical case, that the second term of the pressure is proportional to the first one such that,

$\frac{\mathrm{d}\left[\Psi_{A}^{2} \xi^{2} c^{2}\right]_{1}}{\mathrm{~d} R}-\left(\kappa+2 m_{1}\right) \frac{\mathrm{d}\left[\Psi_{A}^{2} \xi^{2} c^{2}\right]_{0}}{\mathrm{~d} R}=0$.

We deduce from the previous equation that the quantity $\epsilon$, which is defined by

$\epsilon(R) B_{\star}^{2}=\left[\Psi_{A}^{2} \xi^{2} c^{2}\right]_{1}-\left(\kappa+2 m_{1}\right)\left[\Psi_{A}^{2} \xi^{2} c^{2}\right]_{0}=$ cst.

is a dimensionless constant for all the field lines. To give explicitly $\left[\Psi_{A}^{2} \xi^{2} c^{2}\right]_{0}$ and $\left[\Psi_{A}^{2} \xi^{2} c^{2}\right]_{1}$, it may be useful to write

$\Psi_{A}^{2} \xi^{2} c^{2}=\Psi_{A}^{2} \frac{(h \gamma \xi c)^{2}}{h^{2}}\left(1-\frac{\left(V^{\hat{\phi}}\right)^{2}}{c^{2}}\right)-\frac{M_{A l f}^{4} B_{\mathrm{p}}^{2}}{h^{2}}$.

Finally the calculation leads to

$$
\begin{aligned}
\epsilon= & \frac{M^{4}}{h_{z}^{2} h_{\star}^{4} G^{2}\left(R^{2}+l^{2}\right)}\left[\frac{h_{z}^{2} F^{2}\left(R^{2}+l^{2}\right)}{4 R^{2}}-\frac{R^{2}}{\left(R^{2}+l^{2}\right)}\right. \\
& \left.-\left(\kappa-2 m_{1}\right) \frac{\left(R^{2}+l^{2}\right)}{G^{2}}\right]-\frac{v^{2}\left(2 e_{1}-2 m_{1}+\delta-\kappa\right) R}{h_{z}^{2}\left(R^{2}+l^{2}\right)} \\
& -\frac{v^{2} l^{2} R G^{2}}{h_{z}^{2}\left(R^{2}+l^{2}\right)^{3}}+\frac{2 \lambda^{2}}{h_{z}^{2}}\left(\frac{\Lambda^{2} N_{B}}{D}+\frac{\bar{\omega}_{z}}{\lambda}\right)+\lambda^{2}\left(\frac{\Lambda N_{V}}{h_{\star} G D}\right)^{2} .
\end{aligned}
$$

This equation is similar to Eq. (71) in Meliani et al. (2006) and can be interpreted the same way. The parameter $\epsilon$ measures the efficiency of the magnetic rotator to collimate the flow. At the outflow base, $\epsilon$ is the relative difference of the transverse variation of internal energy that is simply the exchange of work done by the macroscopic forces. As this is perpendicular to the flow axis, this means that $\epsilon$ really measures the transverse force which collimates the flow and mainly its magnetic component.

We note that the quantity $-2 m_{1}$ appears twice in Eq. (90). First, it is associated with $\kappa$, having a similar effect to the nonspherically symmetric pressure in the term which is given as a factor of $M^{4}$. Second, it is associated with $2 e_{1}$ in the term corresponding to the excess or the deficit of the gravitational energy not compensated by the thermal driving at the base of the jet.

To conclude we can also derive the magnetic collimation efficiency in a different form; after some calculations, we can write

$$
\begin{aligned}
\epsilon & =-\left.\frac{v^{2} h_{\star}^{4}}{\mu \gamma_{z}^{2} h_{z}^{2}} \frac{\partial}{\partial \alpha} \ln \left(\frac{P-P_{0}}{\rho_{0} \xi}\right)\right|_{\alpha=0} \\
& =h_{\star}^{2} \frac{v^{2}}{\mu}\left(1-\frac{\mu}{v^{2}}\right) \frac{\xi_{z}^{2}}{\xi_{\star}^{2}}\left(\left.\frac{\partial}{\partial \alpha} \ln \left(\rho_{0} \xi\right)\right|_{\alpha=0}-\kappa\right) .
\end{aligned}
$$

This new relation brings a link between the total enthalpy on the axis and its logarithmic variation with $\alpha$. In particular, the sign of $\epsilon$ seems to connect the balance between logarithmic variation of total enthalpy per unit of volume and the meridional increase of the pressure. The factor indicates that $|\epsilon|$ probably tends to decrease for solutions which reach ultra-relativistic speed.

\section{Methodology for obtaining solutions}

\subsection{Numeral integration}

Appendix C gives the coupled ordinary differential Eqs. (C.1), (C.5), (C.6) for the four quantities of the Alfvén number, dimensionless radius, expansion factor, and pressure, $\left(M^{2}, G^{2}, F, \Pi\right)$.
Details on the method and numerical techniques for the integration of these differential equations of the model system can be found in Sauty \& Tsinganos (1994) and Meliani et al. (2006). Briefly, by using a Runge-Kutta scheme we start integrating from the Alfvén surface using the continuity relations. Integrating upwind by adjusting the value of the slope of the derivative of the Alfvén number at the Alfvén transition, the unique value of $F_{\star}$ can be found that allows the crossing of the modified slow magnetosonic surface, for a given value of the pressure at the Alfvén transition $\Pi_{\star}$. Then, integrating downwind, the value of $\Pi_{\star}$ can be further adjusted. After several iterations of upstream and downstream integrations, the program automatically finds the solution that crosses all critical points, a proxy that the asymptotic pressure converges towards the demanded value. In particular, in the following Sections we have selected only solutions with the minimum possible value of $\Pi_{\star}$, the so-called limiting solutions (see Sauty et al. 2004). We either have a collimated jet where, at infinity, $\Pi_{\infty}$ is minimum, or a conical wind where, at infinity, $\Pi_{\infty}$ is zero. If necessary, we can always add a constant value to the pressure $P_{0}$ to ensure that the pressure is positive everywhere in the flow.

In the following, we only outline briefly the points which differ from previous related studies for building our model in the framework of general relativistic MHD in a Kerr metric. Some interesting spine jet solutions, both for their properties close to the black hole and at large distance, are presented in Sect. 5. The presented solutions depend on a number of parameters and a systematic parametric study of the model is postponed for a following paper.

\subsection{Alfvén regularity conditions}

The regularity conditions at the Alfvén transition $(R=1)$ for the azimuthal components and enthalpy have already been discussed in Sect. 2.5. In order for the field lines to not have a kink at the Alfvén transition (i.e., $F$ is a continuous function across $R=1$ ), we impose an extra regularity condition on the transfield equation giving the four physical quantities $\left(M^{2}, G^{2}, F, \Pi\right)$ at $R=1$ (Tsinganos \& Trussoni 1991, Sauty \& Tsinganos 1994). In other words, similarly to the classical model Sauty \& Tsinganos (1994), we should take appropriately into account this regularity condition at $R=1$, which gives the ratio $\tau$ which is involved in the magnetic toroidal component,

$$
\begin{aligned}
\tau & =\left.\frac{N_{B}}{D}\right|_{\star}=\frac{\left.\frac{\mathrm{d} N_{B}}{\mathrm{~d} R}\right|_{\star}}{\left.\frac{\mathrm{d} D}{\mathrm{~d} R}\right|_{\star}} \\
& =\frac{h_{\star}^{2}\left(\frac{2}{1+l^{2}}-F_{\star}\right)-\frac{\mu\left(1-l^{2}\right)}{\left(1+l^{2}\right)^{2}}-\frac{h_{\star} l \sqrt{\mu} v}{\lambda} \frac{l^{2}-3}{\left(1+l^{2}\right)^{3}}}{p-\frac{\mu\left(1-l^{2}\right)}{\left(1+l^{2}\right)^{2}}},
\end{aligned}
$$

where $p$ is the slope of the square of the Alfvén number at the Alfvén transition, and

$$
\left.p \equiv \frac{\mathrm{d} M^{2}}{\mathrm{~d} R}\right|_{\star} .
$$

More specifically, the expansion factor $F$ is determined by Eqs. (C.1) and (C.3). Also in Eq. (C.3) are the ratios of $N_{V}$ and $N_{B}$ with $D$. Furthermore, $D$ appears in the denominator with a power higher by one than the powers of $N_{V}$ and $N_{B}$ in the 
numerator. Hence, in order that $\mathrm{d} F / \mathrm{d} R$ does not diverge and the slope of $F(R)$ is continuous across $R=1$ we require that $\left.\mathcal{N}_{F} \cdot D\right|_{\star}=0$ such that $\mathrm{d} F / \mathrm{d} R$ is finite at $R=1$ wherein we have $0 / 0$. Thus, near the Alfvén transition, we may define the function $\mathcal{N}_{F} \cdot D \sim \mathcal{P}\left(M^{2}, G^{2}, F, \Pi\right)$. Then, in order to avoid a singularity at the Alfvén transition, a necessary condition is to choose $p, F_{\star}, \Pi_{\star}$ such that this function is zero, that is, $\mathcal{P}\left(M^{2}=h_{\star}^{2}, G^{2}=1, F_{\star}, \Pi_{\star}\right)=0$, and thus $\left.\mathcal{N}_{F} \cdot D\right|_{\star}=0$. After some algebra we finally get a second degree polynomial for $F_{\star}$, namely,

$\mathcal{A}(p) F_{\star}^{2}+\mathcal{B}(p) F_{\star}+C\left(p, \Pi_{\star}\right)=0$,

with

$$
\begin{aligned}
\mathcal{A}(p)= & \lambda^{2} h_{\star}^{4}+\frac{h_{\star}^{2}}{4}\left(p-\frac{\mu\left(1-l^{2}\right)}{\left(1+l^{2}\right)^{2}}\right)^{2} \\
\mathcal{B}(p)= & {\left[\frac{1}{2}\left(\frac{\mu\left(1-l^{2}\right)}{\left(1+l^{2}\right)^{2}}-p\right)^{3}\right.} \\
& -2 \lambda^{2} h_{\star}^{2}\left(p+\frac{2}{1+l^{2}}-\frac{2 \mu\left(1-l^{2}\right)}{\left(1+l^{2}\right)^{2}}\right. \\
+ & \left.\left.\frac{l \sqrt{\mu} v h_{\star}}{\lambda} \frac{3-l^{2}}{\left(1+l^{2}\right)^{3}}\right)\right] \\
C\left(p, \Pi_{\star}\right)= & \lambda^{2}\left(p+\frac{2 h_{\star}^{2}}{1+l^{2}}-\frac{2 \mu\left(1-l^{2}\right)}{\left(1+l^{2}\right)^{2}}\right. \\
& \left.+\frac{l \sqrt{\mu} v h_{\star}}{\lambda} \frac{3-l^{2}}{\left(1+l^{2}\right)^{3}}\right)^{2} \\
& +\left(\kappa \Pi_{\star}-\frac{1}{\left(1+l^{2}\right)^{2}}-\frac{2 \lambda^{2} \mu}{v^{2}}\right. \\
& \left.-2 \lambda^{2}-\frac{l^{2}\left(2 \mu+v^{2}\right)}{\left(1+l^{2}\right)^{3}}\right)\left(p-\frac{\mu\left(1-l^{2}\right)}{\left(1+l^{2}\right)^{2}}\right)^{2} .
\end{aligned}
$$

In this way, the regularity condition at the Alfvén transition is automatically satisfied and no more constraints are needed at $R=1$.

\subsection{Effect of a nonspherical Alfvén number in a Schwarzschild metric}

To illustrate our model, we present the following two solutions, which are built in the framework of the Schwarzschild metric. The first one corresponds to a solution of a model presented in Meliani et al. (2006), in which $m_{1}=0$.

The chosen values of the other parameters are $\lambda=1.0$, $\kappa=0.2, \delta=1.2, v=0.8, \ell=0, \mu=0.1, e_{1}=0$. We compare this solution to a solution with the same parameters but by keeping the value of $m_{1}$ given by Eq. (61), $m_{1}=-0.078$. In both solutions, the value of $\Pi_{\star}$ is the minimum value of the limiting solution. Such solutions have the minimum amplitude of oscillations in the jet.

In Fig. 2 the radial velocity on the polar axis is compared for the two solutions, while field lines in the poloidal plane are plotted in Fig. 3. We note that in Eqs. (C.1) and (C.4) and Eqs. (C.1) and (C.3), giving the plasma acceleration and the variation of the expansion factor with the radius $R$, respectively, there are several terms proportional to the factor $\left(\kappa-2 m_{1}\right)$. As $m_{1}$ is always negative, it is evident that $-m_{1}$ effectively increases the transverse pressure gradient, which is proportional to $\kappa$. In other words, the effect of $-m_{1}$ is similar to the effect of $\kappa$ which enforces collimation for $\kappa>0$. Hence, taking into account a nonspherical

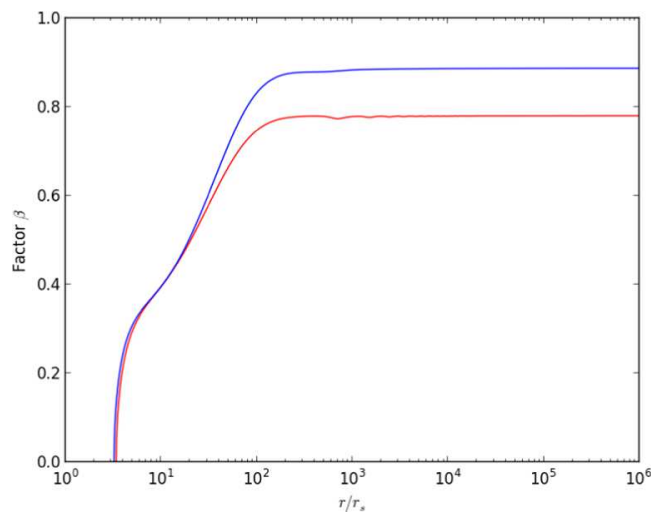

Fig. 2. Evolution of the radial velocity along the polar axis for solutions in a Schwarzschild metric, with $m_{1}=0$ (blue) and $m_{1}=-0.078$ (red). The second case has a smaller terminal velocity.

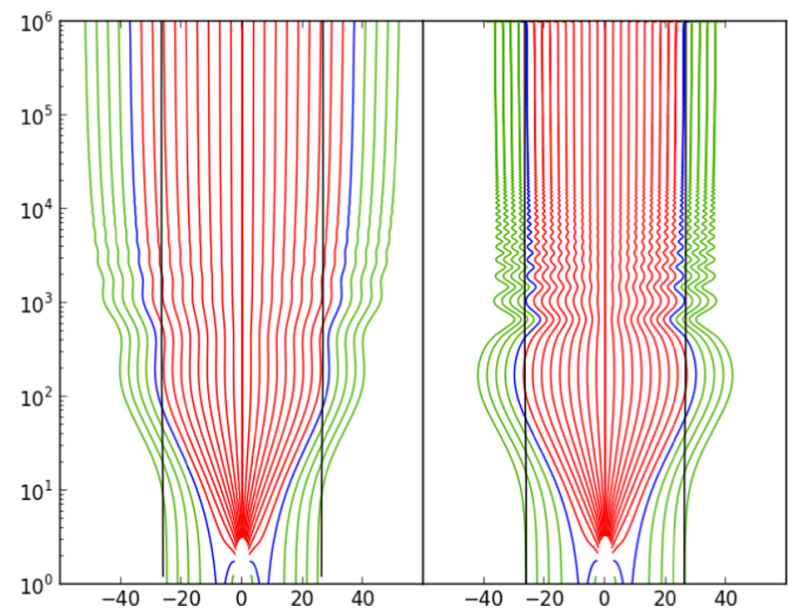

Fig. 3. Field lines for a solution in a Schwarzschild metric, with parameters $\lambda=1.0, \kappa=0.2, \delta=1.2, v=0.8, \ell=0, \mu=0.1, e_{1}=0$ and $m_{1}=0$ (left) and $m_{1}=-0.078$ (right). We note that the case $m_{1}=-0.078$ corresponds to a more tightly collimated jet. Lengths are in units of the Schwarzschild radius. The red lines are connected to the magnetosphere of the central object while the green lines are connected to the disk. The separating line is in blue and the light cylinder in black.

Alfvén number $\left(m_{1} \neq 0\right)$ introduces an extra collimation force which explains why the second solution with a nonzero $m_{1}$ is more collimated. Indeed, the width of the jet at infinity over its value at the base, $G_{\infty} / G_{0}$, decreases from 13.74 to 9.42 , which is similar to an increase of $\kappa$ and this fact can be checked directly by looking at the poloidal field line's shape shown in Fig. 3.

Since more tightly collimated solutions have a smaller super-Alfvénic acceleration, the second solution reaches a lower Lorentz factor asymptotically. Thus, similarly to $\kappa$, the introduction of a negative $m_{1} \neq 0$ leads to a decrease of the velocity because of the tighter collimation. The higher collimation reduces the pressure gradient along the axis, which in turn decreases the acceleration due to pressure driving on large distances (see Sauty et al. 2004).

Additionally, $m_{1}$ appears within the term $\left(\kappa+2 m_{1}-\delta-\right.$ $2 e_{1}$ ) which appears in the plasma acceleration function $\mathcal{N}_{M^{2}}$ in Eqs. (C.1) and (C.4) and the function $\mathcal{N}_{F}$ determining the expansion factor $F$ in Eqs. (C.1) and (C.3). The first three terms $\left(\kappa+2 m_{1}-\delta\right)$ arise from the variation across the field lines of the heat content $P / \rho$ with $\alpha$, that is, $\partial / \partial \alpha\left\{\left[\Pi(R) M^{2}(R)\right](1+\kappa \alpha)(1+\right.$ $\left.\left.m_{1} \alpha\right)^{2} /(1+\delta \alpha]\right\} \simeq\left[\Pi(R) M^{2}(R)\right]\left(\kappa+2 m_{1}-\delta\right)$, while the fourth 
Table 1. Set of parameters used for the four selected solutions in the Kerr metric.

\begin{tabular}{ccccccc}
\hline \hline & $\lambda$ & $\kappa$ & $\delta$ & $v$ & $\mu$ & $l$ \\
\hline K1 & 1.0 & 0.2 & 2.3 & 0.9 & 0.1 & 0.05 \\
\hline K2 & 1.0 & 0.2 & 1.35 & 0.46223 & 0.1 & 0.05 \\
\hline K3 & 1.2 & 0.005 & 2.3 & 0.42 & 0.08 & 0.024 \\
\hline K4 & 0.0143 & 1.451 & 3.14 & 0.8 & 0.41 & 0.15 \\
\hline
\end{tabular}

Notes. K1 is the solution displayed in Figs. 4-6 (blue line). Solution K2 is displayed in Figs. 6 (red line) and 7, while solution $\mathbf{K 3}$ is displayed in Figs. 12 and 13. Finally, solution K4 is shown in Figs. 14 and 15.

term $e_{1}$ is proportional to the variation of the total energy $\mathcal{E}$ with $\alpha$. The bigger this term is, the larger the initial acceleration (see Sauty \& Tsinganos 1994), because it is linked with the distribution of the heating which opposes gravity to accelerate the outflow. As the weight of the plasma decreases with the latitude, then the pressure gradient increases along the axis resulting in a larger acceleration close to the base, as explained in Tsinganos \& Sauty (1992). This term decreases rapidly as the Alfvén surface is reached. Thus, it is responsible only for the initial acceleration. With the parameter $m_{1}$ being negative, the second solution is more accelerated between the base and the Alfvén surface. The velocity of the second solution reaches the velocity of the first one at the Alfvén surface. This effect disappears far from the source.

\section{Solutions in a Kerr metric}

In the following, we discuss four different solutions in a Kerr metric to illustrate the present model. A more detailed parametric study is postponed to a following paper. For the purposes of the present paper, we show three cylindrically collimated solutions with high asymptotic Lorentz factor, typical of AGNs and GRBs. Those solutions cross the "light cylinder" and are sorted with increasing magnetic collimation efficiency parameter $\epsilon$. We also exhibit a conical solution crossing the "light cylinder" with high Lorentz factor and strongly negative $\epsilon$, something that was not possible with the previous relativistic meridionally self-similar solutions.

In order to get a Lorentz factor as high as possible in the asymptotic part of the collimated part of the jet, we know from the study of the classical solutions that among all cylindrical solutions, the limiting solutions with the lowest value of $\Pi_{\star}$ reach the highest terminal velocity. These solutions are the so called limiting solutions in Sauty et al. (2002). As $\Pi_{\infty}$ is negative for the limiting solutions, we have to add a positive $P_{0}$ value to the pressure. Of course, it is always possible for those cylindrical solutions to have a higher pressure $P_{0}$, but by doing so it also increases the effective temperature, in particular in the asymptotic part. For the same set of parameters, it is also possible to get cylindrical solutions by increasing $\Pi_{\star}$. However, such solutions usually have a strong initial decollimation associated with a peak in both the Lorentz factor and temperature, while the asymptotic jet is decelerated to lower Lorentz factors and smaller radii, a result that we used to interpret the FRI/FRII dichotomy [cf. Meliani et al. (2010)].

Solutions K1 and K2 have been obtained for maximally rotating black holes, that is, $a_{H}$ close to $1\left(a \simeq r_{s} / 2\right)$. In solution $\mathbf{K} 4$ the value of $a_{H}$ has been fixed to $0.73\left(a=0.73 r_{s} / 2\right)$. We do not expect all black holes to be maximally rotating. For
Table 2. Output parameters for the four solutions in the Kerr metric.

\begin{tabular}{ccccc}
\hline \hline & $\epsilon$ & $m_{1}$ & $\Pi_{\star, \lim }$ & $r_{0} / r_{s}$ \\
\hline K1 & -1.76 & -0.062 & 0.826 & 5.72 \\
\hline K2 & -0.04 & -0.234 & 0.216 & 1.57 \\
\hline K3 & 0.55 & -0.326 & 0.189 & 2.55 \\
\hline K4 & -5.84 & -0.004 & 0.255 & 1.39
\end{tabular}

Notes. Those parameters result from the integration of the equations.

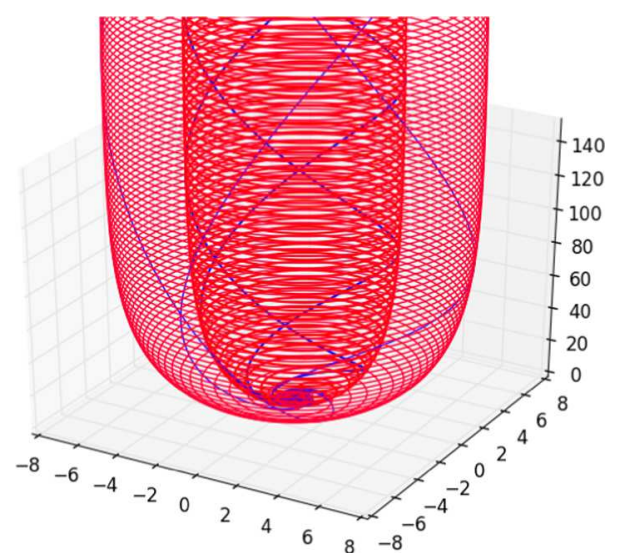

Fig. 4. Three-dimensional (3D) representation of the field lines and streamlines for the thermally collimated solution $\mathbf{K} \mathbf{1}$ at the base of the jet and for two flux tubes. The blue lines correspond to streamlines, the red lines to magnetic field lines. The length is in units of the Alfvén radius, that is, ten times the Schwarzschild radius.

example, in M 87, the dimensionless spin should be above 0.65 (i.e., $a>0.65 r_{s} / 2$ ), (Li et al. 2009) but not too close to one. Other examples can be found and for $\mathbf{K 3}$ we will use the value $a_{H}=0.6\left(a=0.6 r_{s} / 2\right)$ adopted in Mertens et al. (2016) for M 87.

\subsection{A mildly relativistic collimated solution with oscillations (K1)}

The collimated solution K1 corresponds to an over-pressured outflow $(\kappa \geq 0)$ in a Kerr metric. As $\epsilon \leq 0$, the collimation of the jet is not fully magnetic but it has a significant contribution by the gas pressure, at least during the phase of strong acceleration up to $\simeq 30 r_{s}$. In this solution, field lines are strongly oscillating compared to the two previous solutions in the Schwarzschild metric. The outflow undergoes a series of strong oscillations connected to the balance between the toroidal magnetic tension and the decollimation forces (centrifugal and electric forces and transverse pressure gradient) of the plasma.

The significant contribution of the transverse pressure gradient also explains these strong oscillations in the flow, as is also shown in the classical solutions. The parameters of this solution are displayed in the first row K1 of Table 1 and the output values of $m_{1}$ and $\epsilon$ in Table 2. We note that $m_{1}=-0.062$ is a relatively small value, which clearly indicates that the Alfvén surface is almost spherically symmetric in this case. As a consequence, the light cylinder is relatively far from the jet axis. Most of the central field lines (see the inner 5 to 7 central red lines in Fig. 5) remain within the "light cylinder", which means that despite the important role of the magnetic field in the collimation, the jet is pressure or enthalpy driven in the relativistic case. However, unlike in the classical solutions, the electric field is the 


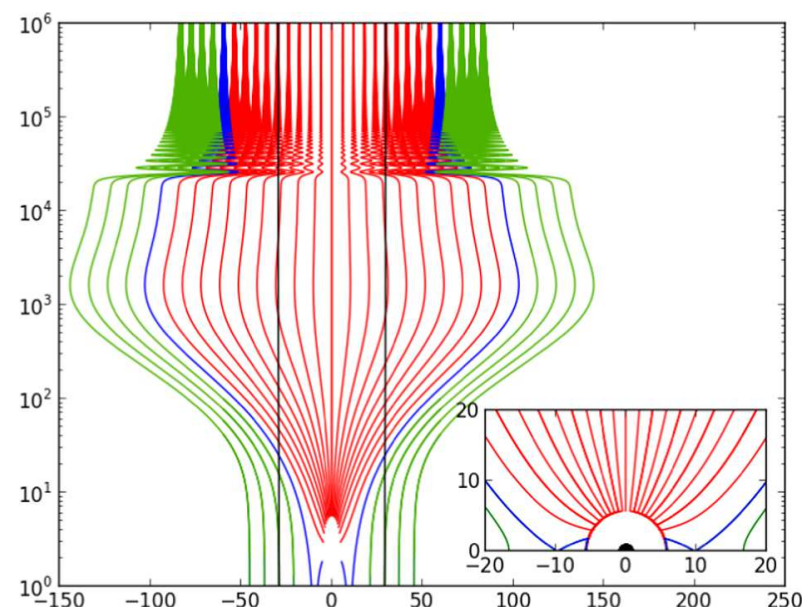

Fig. 5. Poloidal field lines and "light cylinder" for the thermally collimated solution K1, for $\lambda=1.0, \kappa=0.2, \delta=2.3, v=0.9, \mu=0.1$, $\ell=0.05, e_{1}=0$. The length unit is the Schwarzschild radius.

dominant decollimating force for the lines that cross the "light cylinder". This decollimation and expansion after the Alfvén surface is associated with a strong pressure gradient yielding a strong acceleration of the jet in the super-Alfvénic regime. More details on this will be given in the following solution. The pressure gradient is the gas pressure gradient close to the axis but assisted by the toroidal magnetic pressure outside the "light cylinder". This is similar to superfast flows in radially selfsimilar models for disk winds (see Vlahakis \& Königl 2003a and Vlahakis \& Königl 2003b). Moreover, in the relativistic regime the inertia increases faster when the flow is accelerated such that the collimation from the magnetic field is delayed to larger distances.

In Fig. 5 we see that the expansion after the Alfvén surface is strong and leads to a late acceleration of the flow. After the large expansion, the jet recollimates smoothly and consequently decelerates slightly because of the compression.

In Fig. 4, we clearly see that there is a strong azimuthal magnetic field, although the scale of the figure tends to exaggerate this phenomenon.

The Lorentz factor $\gamma$ of this solution reaches a relatively small value around 3.7, typical of less powerful AGN jets like some of the FRI radio-galaxies.

\subsection{A highly relativistic collimated solution with oscillations (K2)}

The solution $\mathbf{K} 2$ is collimated and has an extremely high Lorentz factor, which may be typical of GRBs. This K2 model corresponds to the values of the parameters given in the second line of Table 1 , that is, $\lambda=1.0, \kappa=0.2, \delta=1.35, v=0.46223$, $\ell=0.5, \mu=0.1, e_{1}=0$ and the second line of Table 2 for the output parameters, $m_{1}=-0.234$ and $\boldsymbol{\epsilon}=-0.04$.

For this model, the outflow starts very close to the black hole horizon at $r_{0}=1.57 r_{s}$, (see Fig. 7), and thus at the base of the jet the effects of general relativity play an important role. The final velocity is highly relativistic, as is shown in Fig. 6.

The parameter $v$ of the $\mathbf{K} \mathbf{2}$ solution is accurately adjusted (to the fifth digit), so as to obtain a rather high Lorentz factor (larger than 100). This proves the versatility of the model which handles any magnitude of Lorentz factors. In order to obtain such high Lorentz factors, we must carefully tune the parameter directly linked to gravitation, $v$, as mentioned above. The same parameter

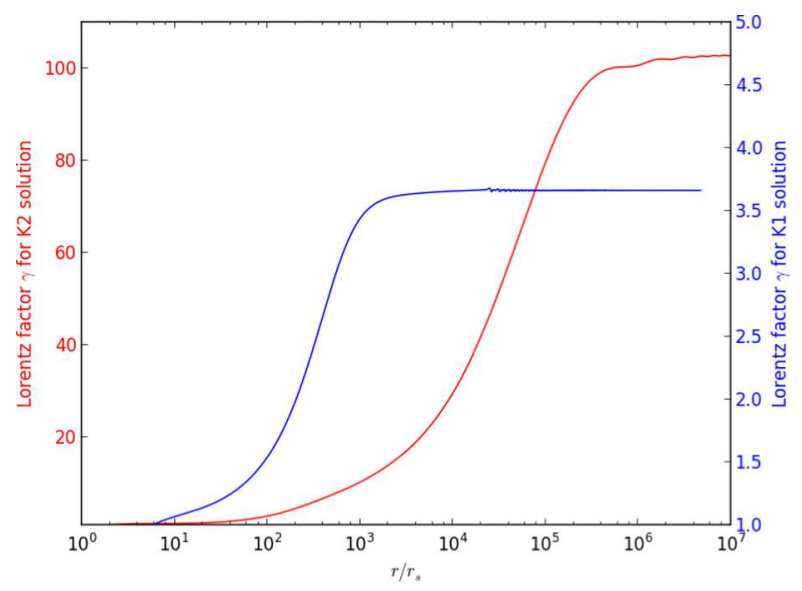

Fig. 6. Lorentz factor $\gamma$ for the K1 (blue line) and K2 (red line) solutions. Distances are given in Schwarzschild radius units.

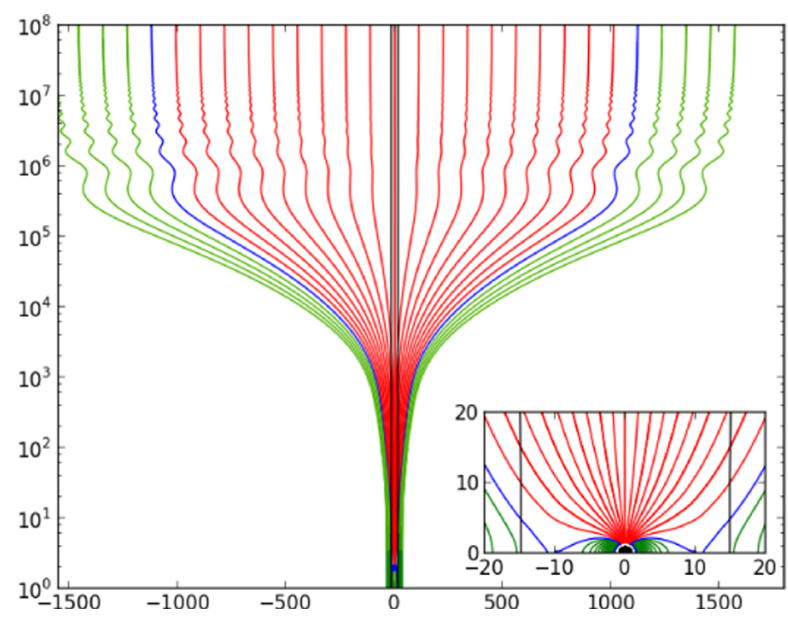

Fig. 7. Poloidal field lines and "light cylinder" for the $\mathbf{K} 2$ solution, that is, for $\lambda=1.0, \kappa=0.2, \delta=1.35, v=0.46223, \mu=0.1, \ell=0.05$, $e_{1}=0$. Distances are given in Schwarzschild radius units.

is also responsible for the thermal acceleration in the classical model (Sauty \& Tsinganos 1994).

In Figs. 8 and 9 we plot the forces along and perpendicular to a field line defined by $\alpha=0.01 \alpha_{\text {lim }}$ where $\alpha_{\text {lim }}$ is the dimensionless magnetic flux between the inner jet and an external accretion disk wind.

The strong decollimation associated with the slow acceleration enhances the electric force as in the previous solution K1. This can be seen in Fig. 9. However due to the higher rotation here, more field lines cross the light cylinder, which is very close to the axis, such that the decollimation from the electric field is much stronger in this solution.

The feedback of this strong electric field is to further increase the decollimation beyond the Alfvén surface at large distances. Again, the large expansion increases the pressure and enthalpy gradient as seen in Fig. 8. Thus, the pressure force increases, resulting in a very long acceleration phase up to $10^{6} r_{s}$. Thus, the thermal acceleration becomes very efficient and the plasma asymptotically reaches an extremely high Lorentz factor. Indeed, the jet radius increase of this solution is also very high with an expansion factor $G_{\infty} / G_{0} \simeq 1300$, as can be seen in Fig. 7 .

The value of $\epsilon$ is still negative but very close to zero $(\epsilon=-0.04)$. As in the classical case, this means the magnetic efficiency to collimate the flow is higher in this model at larges 


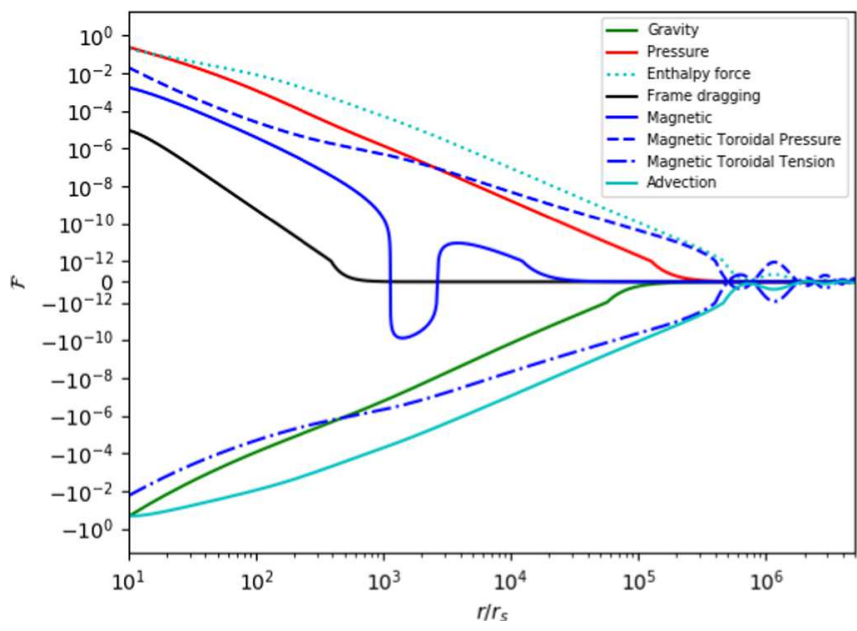

Fig. 8. Longitudinal forces, that is, along the field line, for the K2 solution, along the line $\alpha=0.01 \alpha_{\mathrm{lim}}$. Distances are given in Schwarzschild radius units.

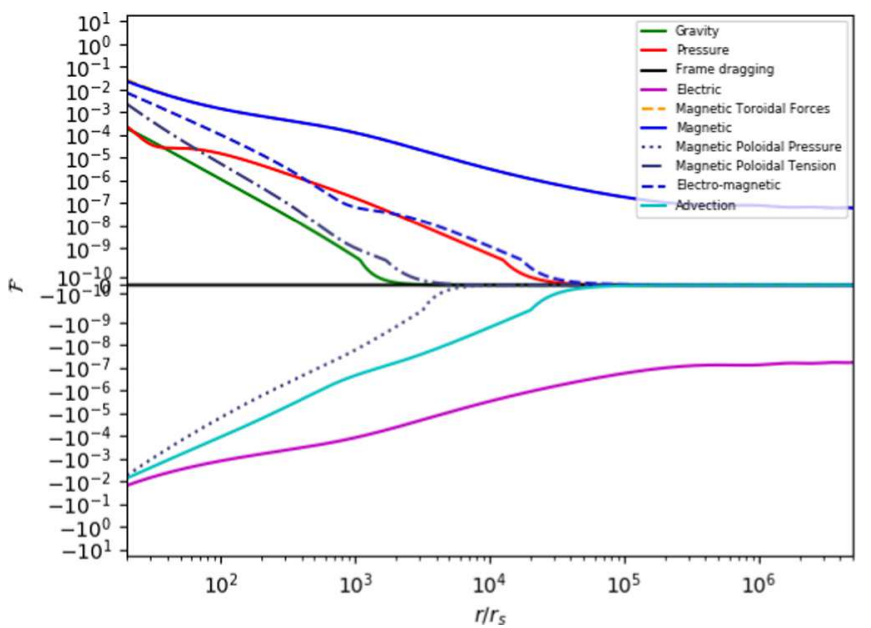

Fig. 9. Transverse forces, that is, perpendicular to the field line, for the K2 solution, along the line $\alpha=0.01 \alpha_{\mathrm{lim}}$. We see that the Lorentz force is collimating and is balanced by the electric force that decollimates. Distances are given in Schwarzschild radius units.

distances. However the decollimating force that ensures the equilibrium is no longer the centrifugal force or the pressure gradient but the electric force on the lines that cross the "light cylinder". This is a specific feature of relativistic jets.

To further analyze the jet acceleration we may calculate the contribution of the different components of the total energy and the conversion of the magnitude of each component to another form along the streamlines. First, we want to obtain a physically acceptable heating term which goes to zero at infinity along the axis of the flow. By defining the enthalpy in Eqs. (18) and (19) and its analytical expression in our model in Eq. (34), we may fix a streamline limiting the zone where the pressure is positive. Once this field line has been chosen, for example, at $\alpha=0.4 \alpha_{\text {lim }}$, the dimensionless pressure $P_{0} / \rho_{\star} c^{2}$ can be calculated. In the particular case presented here, we have taken $P_{0} / \rho_{\star} c^{2}=4.3 \times 10^{-7}$ . The pressure $P_{0}$ in Eq. (54) is chosen such that the gas pressure is equal to zero, when $\Pi(R)$ reaches its minimum value. For some value of $\alpha$, the term $P_{0}=P_{0}(\alpha)=-\left[B_{\star}^{2} / 8 \pi\right] \Pi_{\min }(1+\kappa \alpha)$ will be too large to ensure that $Q$ goes to zero at infinity along the axis. Indeed, $\alpha$ can take any value below a maximum $\alpha_{\max }$. We can consider that the solution can be valid only in the region

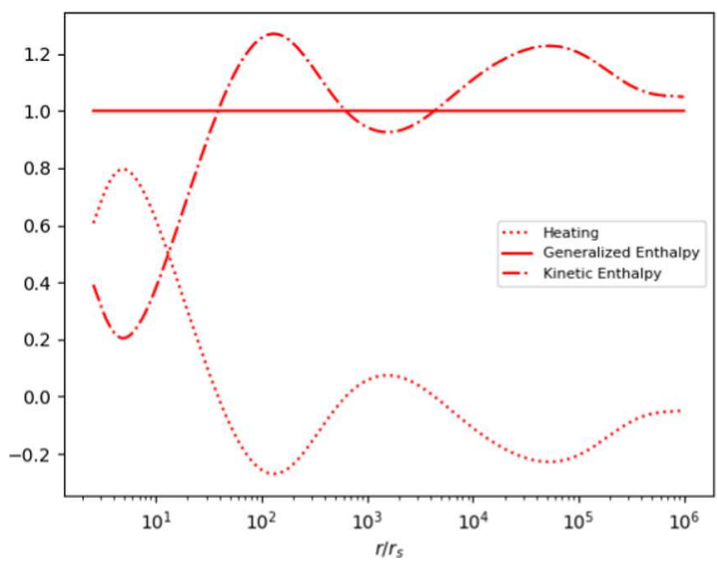

Fig. 10. Relative normalized contribution to the total energy of the kinetic enthalpy $h \gamma \xi_{k}$ and the external heating distribution $h \gamma Q / c^{2}$ along the axis.

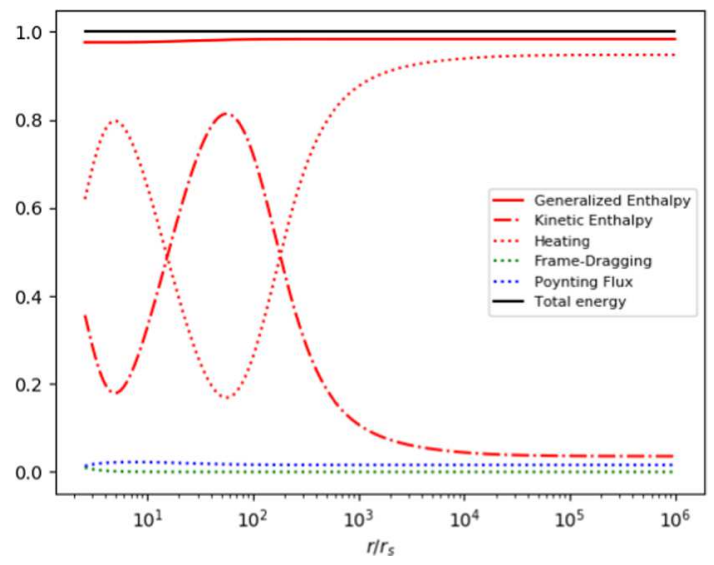

Fig. 11. Relative normalized contribution to the total energy of the kinetic enthalpy $h \gamma \xi_{k}$ and the external heating distribution $h \gamma Q / c^{2}$ along the streamline $\alpha=0.05 \alpha_{\text {lim }}$

wherein the pressure is positive. When $\alpha$ is fixed and $P_{0} / \rho_{\star} c^{2}$ is deduced, we are able to calculate $\xi_{\star}$. In this particular case, a value $\xi_{\star} \simeq 78$ is chosen.

Figure 10 shows the normalized total energy on the axis $\mathcal{E} / c^{2}=h \gamma \xi$, the kinetic component $h \gamma \xi_{K}$ and the external heating $h \gamma Q / c^{2}$. We find a decrease of the external heating and a related increase of the kinetic part. Thus, the kinetic enthalpy represents the major component up to $r=10^{2} r_{s}$.

Figure 11 shows the same energetic distribution, but on a streamline with $\alpha=0.05 \alpha_{\text {lim }}$. Out of the polar axis, there are extra energetic components, such as the frame-dragging and the Poynting fluxes. Both these energetic contributions are very small on this field line, as compared to the total energy. Hence, the jet is enthalpy-driven from the axis right up to the limiting line. We also note that at the base of the jet, the frame-dragging energy is of the same order as the Poynting flux. Contrary to the energetic distribution along the axis, the external heating constitutes the larger part of energy at infinity. While along the axis a high value of $\gamma_{\infty} \simeq 100$ is obtained, the Lorentz factor at infinity on this particular line is $\gamma_{\infty} \simeq 3.6$. Hence, since the acceleration of the plasma and the resulting final flow speed at infinity on this particular field line are small, the external heating is not consumed when accelerating the flow and therefore it is left unused at infinity, contrary to what happens along the axis; Fig. 10. 


\subsection{A mildly relativistic collimated solution without oscillations (K3)}

From VLBI imaging, Mertens et al. (2016) recovered a detailed two-dimensional velocity field in the jet of M 87 at sub-parsec scales. They confirmed the stratification of the flow from the very beginning of the jet and identified a relativistic sheath, that is, an accelerating layer, which is launched from the inner part of the accretion disk at a cylindrical distance of around $5 r_{s}$. Mertens et al. (2016) interpret this outer sheath layer as the internal part of an external disk wind. They also interpret the inner spine jet as a component coming from the internal accretion disk. However, it is not clear that the inner spine necessarily has to be one of the disk wind components. Instead we propose that the inner spine jet originates from the black hole corona and has a higher Lorentz factor. The authors note that this fast inner spine jet cannot be detected in their data because of its lower emissivity compared to that of the sheath layer or because its speed is too high.

We propose here, as an alternative scenario, that the spine beam may originate from the magnetosphere of the black hole, either connected to the black hole itself, as in the BlandfordZnajek-Penrose mechanism, or connected to the inner part of the accretion disk. In the first case, the spine jet would be a leptonic plasma, and in the second it would be a hadronic one. In such a case, we can model the spine jet with our meridionally selfsimilar solutions. We note that unlike Poynting-flux-dominated models, our model is valid on the jet axis.

The Lorentz factor profile inferred by Mertens et al. (2016, see their Fig. 19) supposes that the velocity structure observed in the $43 \mathrm{GHz}$ VLBA maps at a deprojected distance of $z \simeq 10$ mas $\simeq 1300 r_{s}$ is due to the sheath layer. The Lorentz factor of the sheath has a value $\gamma \simeq 2.4$ at $R \simeq 1000 r_{s}$.

From the curve deduced for the spine jet with an approximated MHD disk wind solution, those authors, following Anderson et al. (2003), model the acceleration and collimation of the flow with a Lorentz factor $\gamma \approx 7$. We propose that it is possible to construct a MHD solution along the lines of the model analysed in this paper, by choosing a positive value of $\epsilon \approx 0.5$ and a similar velocity profile. The solution $\mathbf{K 3}$ presented here has a Lorentz factor $\gamma \simeq 5$ at $R \simeq 1000 r_{s}$. By appropriately tuning the parameters, we could obtain even higher Lorentz factors from 7 to 10 , as is observed at the distance of the knot HST1 , where this velocity is observed in the optical band. However, the spine jet is deboosted relative to the sheath and a precise measurement of its Lorentz factor is difficult.

Solution K3 with a maximum Lorentz factor on the axis $\gamma \simeq 5.5$ has an asymptotic spine jet radius $G_{\infty} \simeq 20 r_{s}$. At this distance from the axis, the Lorentz factor has dropped to a value $\gamma \simeq 2.4$ consistent with the radius and the Lorentz factor at the inner observed distance of the outer sheath jet of Mertens et al. (2016). Thus, our solution may model the initial spine jet inside the sheath layer. However, to confirm that, we need to use this initial solution in simulations similar to those in Hervet et al. (2017). The spine jet/sheath jet interaction will probably produce shocks and rarefaction waves that may further accelerate the jet.

Therefore, although we can obtain such a type of collimated solution for different sets of the parameters, we focus here on the specific solution $\mathbf{K 3}$, where $\lambda=1.2, \kappa=0.005, \delta=2.3$, $v=0.409, \ell=0.024$ and $\mu=0.08$. Compared to the other Kerr solutions studied in this paper, $\lambda$ is higher and $\kappa$ is very small, leading to a solution with a positive value of the magnetic collimation efficiency, $\epsilon=0.55$. Another advantage of this solution is the fact that the pressure depends only very weakly on the magnetic flux function, that is, on a particular field line.

Interestingly, in Mertens et al. (2016), the radius of the base of this spine jet is equal to $r_{0}=2.4 r_{s}$, which is consistent with their model of a disk wind solution, by fixing the jet shape and solving the Bernoulli equation. In our self-similar solution, we have a similar radius for the magnetospheric polar cup, where our jet solution starts. Clearly, this is an alternative scenario.

Moreover, the angular velocity of the field lines anchored in this polar cup above the black hole can be calculated from our parameters,

$$
\begin{aligned}
\Omega_{\star} & =\frac{c \mu}{r_{s}}\left[\frac{\sqrt{\mu} \lambda}{v} \sqrt{1-\frac{\mu}{\left(1+l^{2}\right)}}+\frac{l \mu}{\left(1+l^{2}\right)^{2}}\right] \\
& \simeq 6.2 \times 10^{-2} \frac{c}{r_{s}} .
\end{aligned}
$$

By taking the value of the M 87 distance and the black hole mass, as in Mertens et al. (2016), we can calculate $\Omega_{\star}$ in the context of this K3 solution. This value may directly be compared to the values they deduced in two jet regions from the conservation of total energy and angular momentum fluxes in the approximation of special relativity.

Hence, we find $\Omega_{\star} \simeq 1.03 \times 10^{-6} \mathrm{~s}^{-1}$, a value which is for the spine jet almost the same value as the isorotation frequency of a Keplerian speed at the launching location of the sheath layer. It also corresponds to the initial toroidal velocity of the Blandford \& Payne (1982) mechanism.

Our model corresponds to an alternative configuration, because the spine jet may either originate from the Keplerian disk, in which case it would be hadronic, or form via the generalized Penrose-Blandford-Znajek mechanism. In this second alternative, the jet would be a leptonic beam with an angular frequency proportional to the spin of the central black hole. The Blandford-Znajek mechanism allows us to extract energy from the black hole when $0 \leq \Omega \leq \omega_{\mathrm{BH}}$, with a maximum value for $0.5 \omega_{\mathrm{BH}}$. We note that $\omega_{\mathrm{BH}}$ is, by definition, the angular velocity of ZAMO at the location of the outer event horizon and is given by,

$\omega_{\mathrm{BH}}=\frac{a_{H} c}{r_{s}\left(1+\sqrt{1-a_{H}^{2}}\right)}$,

where $a_{H}$ is the dimensionless spin of the black hole in units of the gravitational radius $r_{s} / 2$. Indeed simulations of such Poynting-dominated and force-free jets (Tchekhovskoy 2015) have shown that the angular speed of a field line anchored in the magnetosphere is about half the black hole angular speed $\omega_{\mathrm{BH}}$. Our value of $\Omega_{\star}$ is one third of $0.5 \omega_{\mathrm{BH}}$ ((Nathanail \& Contopoulos 2014)). In order to determine if the spine jet of our solutions originates from a Keplerian disk or from a black hole via a generalized Penrose-Blandford-Znajek mechanism, we need to solve the MHD equations up stream up to the black hole horizon. Moreover, in order to model the full jet of M 87, a complete MHD simulation including a disk wind and a spine jet must be developed. Something that should be done in the future.

We note that as is already known, at the interface of the spine jet and the sheath layer, a re-collimation shock may occur, producing compression and rarefaction waves which may accelerate the flow (Hervet et al. 2017). For those reasons we have chosen here to adjust the value of $v$ to $v=0.42$, in order to get the solution K3, with a lower Lorentz factor but suppressing completely 


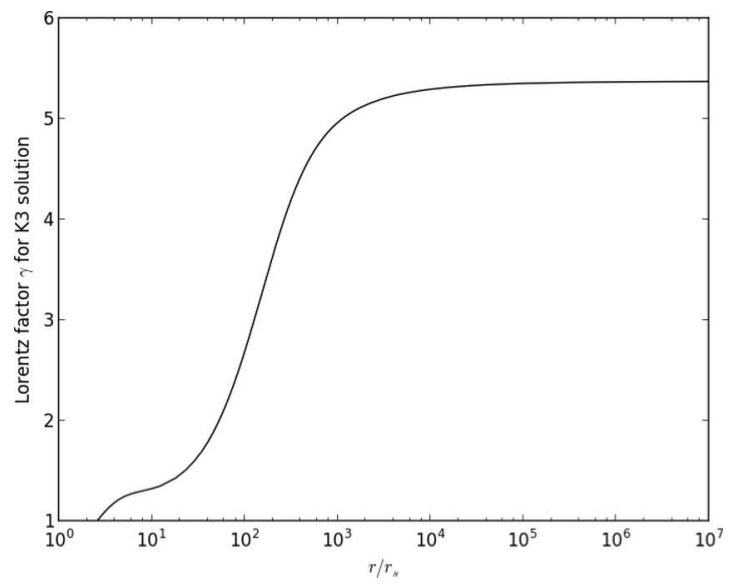

Fig. 12. Lorentz factor for the nonoscillating collimated solution K3.

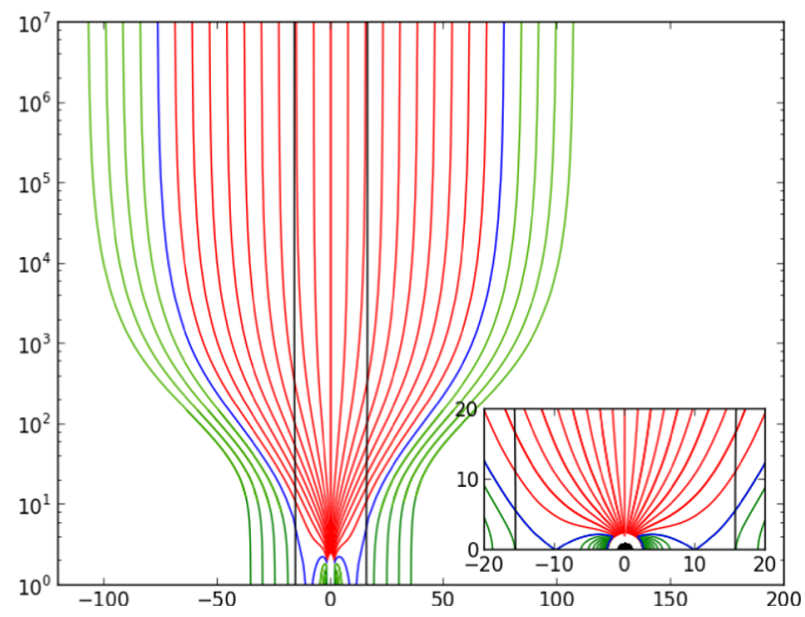

Fig. 13. Poloidal field lines and "light cylinder" for the nonoscillating collimated solution $\mathbf{K 3}$, that is, for $\lambda=1.2, \kappa=0.005$, $\delta=2.3, v=0.42, \mu=0.08, \ell=0.024, e_{1}=0$. Distances are given in Schwarzschild radius units.

the oscillations of the field lines. The value calculated above for $\Omega_{\star}$ is not changed, but radio emission maps, which are obtained for the M 87 jet, will be produced by re-collimation shocks due to the interaction between the fast spine jet and the sheath layer.

As can be seen in Fig. 12 for the $\mathbf{K 3}$ model, the Lorentz factor reaches a nearly constant value at the distance of the $B$ structure observed in the $43 \mathrm{GHz}$ VLBA maps and $\gamma$ is larger than that deduced for the sheath layer by Mertens et al. (2016). As explained above, the interaction between the sheath layer and the spine jet can induce a bulk flow acceleration. However, as we mention, the Lorentz factor $\gamma$ is maximum along the axis and decreases with latitude such that at its outer boundary it matches the sheath layer value.

The values of the parameters of the $\mathbf{K} \mathbf{3}$ model are given in the third line of Tables 1 and 2 . The radius at the base of this spine jet is equal to $r_{0}=2.55 r_{s}$ and the magnetic efficiency to collimate the flow is larger compared to the other two Kerr solutions discussed in this paper, as $\epsilon$ is now positive, which means that the jet is fully magnetically collimated. The field lines displayed for $\mathbf{K 3}$ in Fig. 13 are nearly cylindrical above the equatorial plane, at distances $10^{4} r_{s}$, with a smooth flaring occurring after the Alfvén surface.

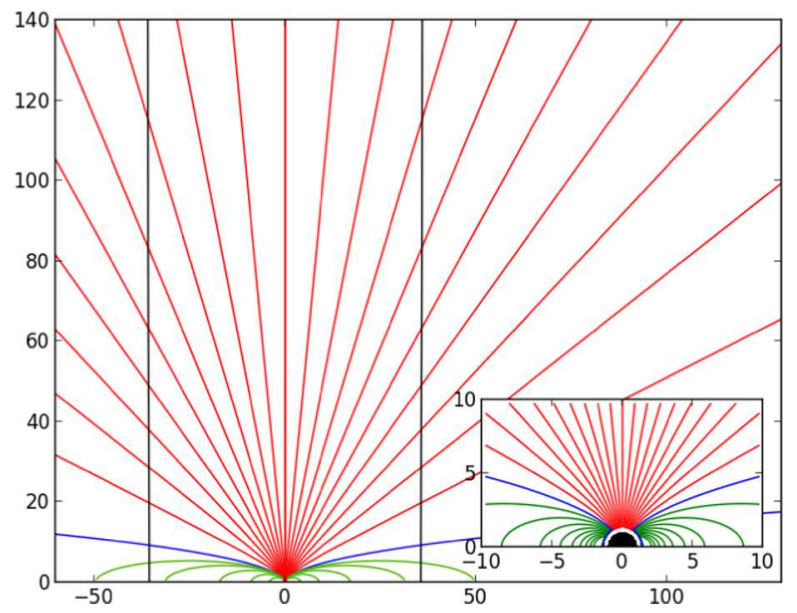

Fig. 14. Poloidal field lines and "light cylinder" for the conical solution K4, that is, for $\lambda=0.0143, \kappa=1.451, \delta=3.14, v=0.8, \mu=0.41$, $\ell=0.15, e_{1}=0$. Distances are given in Schwarzschild radius units.

\subsection{Conical solution}

The parameters of the conical solution $\mathbf{K} \mathbf{4}$ are given in Tables 1 and 2. Such radial solutions could be useful to describe relativistic noncollimated outflows, such as those seen in association with radio-quiet galaxies, such as Seyfert galaxies. Conical solutions could also be useful to model GRBs, wherein an unstable noncollimated relativistic wind may fragment into small pieces under some instabilities. In such cases, the apparent collimation of the GRB would be due to fragmentation - see Meliani \& Keppens (2010); van Eerten et al. (2011).

A conical solution is a solution in which the limiting value of $F$ at infinity is 0 . In this solution, the spherical part of the Mach number diverges, $M \rightarrow \infty$. The same effect occurs for the cylindrical radius of the flow, $G$. To obtain this conical solution, we started with the parameters used for modeling the solar wind, as in Sauty et al. (2005). The magnetic collimation parameter must be strongly negative, $\epsilon /\left(2 \lambda^{2}\right) \leq 0$. We adjust the solution to the relativistic case and increase the velocity by taking a larger value for $\delta$. For the radial solution $\mathbf{K 4}$, the parameters $\lambda$ and $\kappa$ are adjusted as well in order to obtain a terminal Lorentz factor larger than 8. Thus, we find a conical solution $\mathbf{K 4}$ for the following set of parameters: $\lambda=0.0143, \kappa=1.451, \delta=3.14, v=0.8$, $\ell=0.15, \mu=0.41, m_{1}=-0.004$ and $e_{1}=0$.

For this set of parameters we get a much more negative value for the magnetic collimation efficiency parameter, $\epsilon=-5.68$. The solution quickly reaches the conical regime (see Fig. 14), and most of the field lines cross the "light cylinder" plotted as a black solid line. The axial radial velocity profile is plotted in Fig. 15. As can be seen, high Lorentz factors are obtained.

\subsection{Magnetic collimation efficiency versus black hole spin}

As already mentioned, the constant $\epsilon$ is a measure of the efficiency of the magnetorotational forces to collimate the flow. We have studied how $\epsilon$ relates to the black hole spin $a_{H}=2 l / \mu$, keeping unchanged all the other parameters: $\lambda, \kappa, \delta, v$, and $\mu$.

In Fig. 16, we plot $\epsilon$ versus the black hole spin $a_{H}$, for several cases studied in the context of our model. First we note that for solutions with parameters similar to the $\mathbf{K} \mathbf{3}$ solution, referred to here as K3-type solutions, the value of $\epsilon$ is positive, it increases with the black hole spin $a_{H}$ and shows the largest variation in relative magnitude with an absolute total variation equal to 0.05 . 


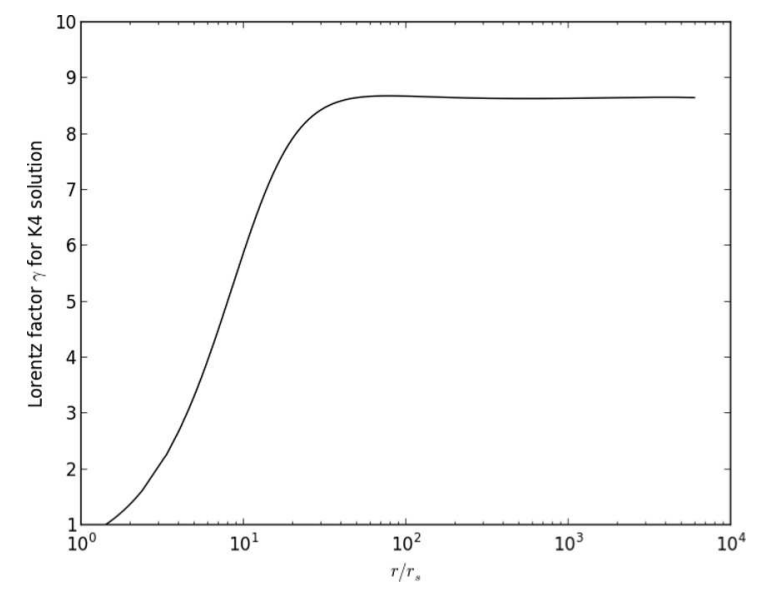

Fig. 15. Lorentz factor for the conical solution K4. Distances are given in Schwarzschild radius units.

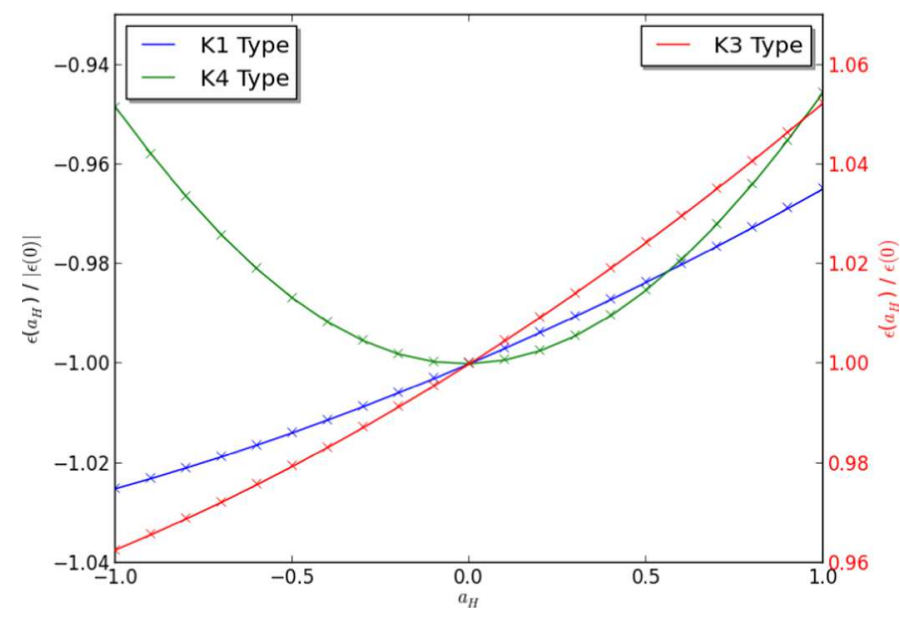

Fig. 16. Variation of the magnetic collimation efficiency parameter $\epsilon$ vs. the black hole spin parameter $a_{H}$ for K1-type, K3-type, and K4type solutions. The value of $\epsilon$ has been normalized by $|\epsilon(0)|$ when it is negative and by $\epsilon(0)$ when it is positive.

On the other hand, for K1-type and K4-type solutions, the values of epsilon are negative. The total variation for K1-type solutions is equal to 0.10 and for the K4-type solutions is equal to 0.25 . We see that $\epsilon$ is increasing with the black hole spin $a_{H}$ for K1-type and K3-type solutions. It also presents a minimum value for $\mathbf{K 4}$ at a black hole spin slightly smaller than $a_{H}=0$. This means that the magnetic collimation efficiency is lower for counter-rotating black holes in relation to their accretion disk for K1-type and K3-type solutions and depends weakly on the spin direction for K4-type ones. However, K4-type solutions are conical, contrary to all other solutions, which are cylindrically collimated. In all cases $\epsilon$ does not vary linearly with the black hole spin $a_{H}$.

This nonlinear variation of epsilon with the black hole spin $a_{H}$ is explained if we try to derive it at the base of the jet where the Alfvénic number is equal to 0 , since some terms of the second order in $l$ cannot be neglected in the following equation.

$$
\begin{aligned}
\epsilon & =\frac{2 \lambda^{2}}{h_{z}^{2}}\left(\frac{\Lambda^{2} N_{B}}{D}+\frac{\bar{\omega}_{z}}{\lambda}\right)+\lambda^{2}\left(\frac{\Lambda N_{V}}{h_{*} G_{0} D}\right)^{2} \\
& -\frac{v^{2}\left(2 e_{1}-2 m_{1}+\delta-\kappa\right) R_{0}}{h_{z}^{2}\left(R_{0}^{2}+l^{2}\right)}-\frac{v^{2} l^{2} R_{0} G_{0}^{2}}{h_{z}^{2}\left(R_{0}^{2}+l^{2}\right)^{3}} .
\end{aligned}
$$

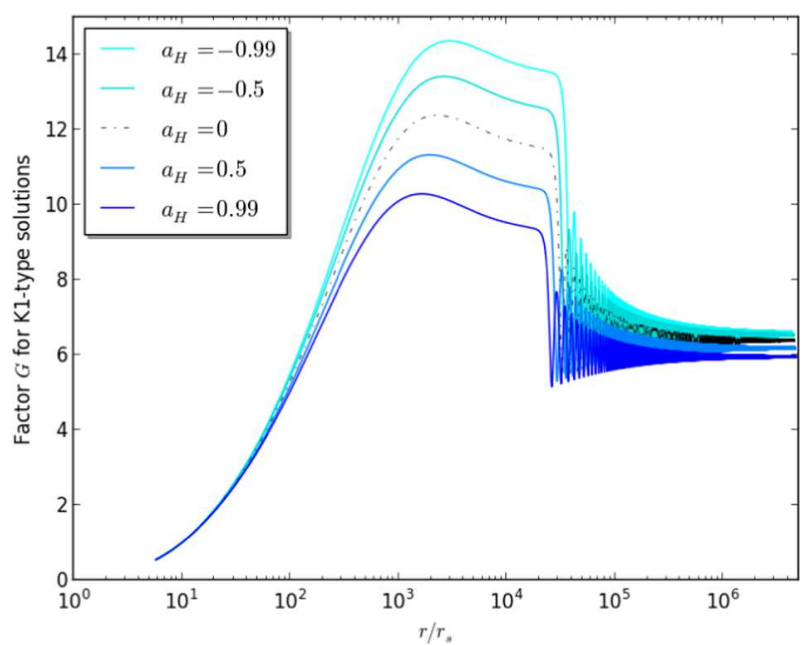

Fig. 17. Cylindrical jet radius normalized to its value at the Alfvén surface, $G$, for K1-type solutions, as a function of the distance along the polar axis, for five different values of the black hole spin $a_{H}$. The function $G$ is equal to 1 at the Alfvén distance $r=10 r_{s}$.

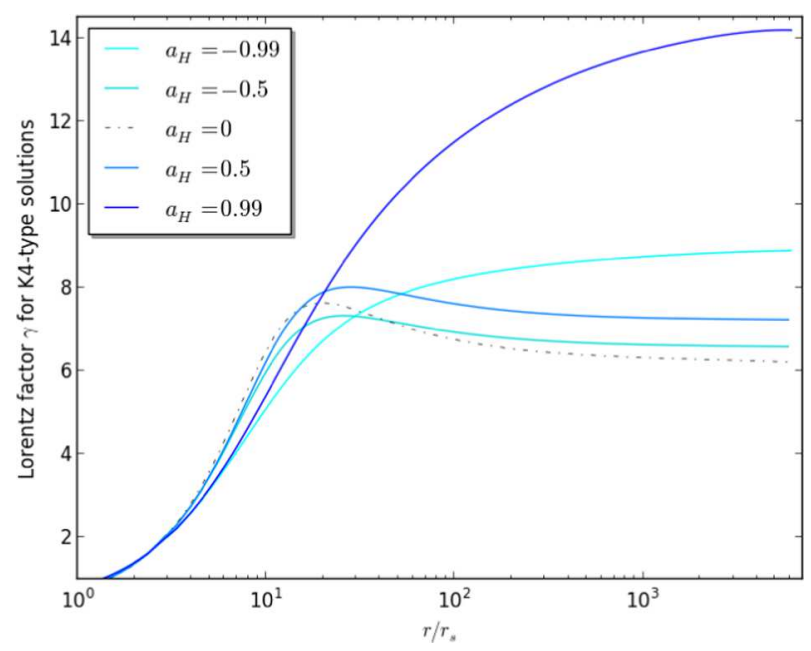

Fig. 18. Lorentz factor $\gamma$ for K4-type solutions when the black hole spin parameter $a_{H}$ varies between -0.99 and 0.99 .

An increase in $\epsilon$ coincides with a decrease in the maximal Lorentz factor for the collimating Kerr solutions, as we will see for K1-type and K3-type solutions. This explains why it was not possible to obtain physical solutions by decreasing $l$ for K2-type solutions. For this second Kerr solution, we performed a fine tuning of the parameters to obtain the largest possible Lorentz factor at large distances from the maximally rotating black hole. Then, decreasing $l$ leads automatically to exceed the value of the speed of light, $c$, for the polar velocity at some distance in the jet. The acceleration phase does not vary for K1-type and K3-type solutions with $l$ except immediately before $R=500$ where the Lorentz factor reaches a plateau. The value of the maximum $\gamma$ increases when $l$ decreases.

For the two collimated solutions $\mathrm{K} \mathbf{1}$ and $\mathbf{K 3}$, there is a clear effect of collimation induced by the rotation of the black hole. In Fig. 17 we plot for five values of the black hole spin the evolution of the factor $G$ along the jet, that is, the ratio of the jet cylindrical radius divided by its value at the Alfvén surface. From this plot it can be seen that the maximum of the radius of the jet is reached at different distances, as the spin varies, and decreases when $a_{H}$ increases. The same trend is observed for the terminal jet radius 


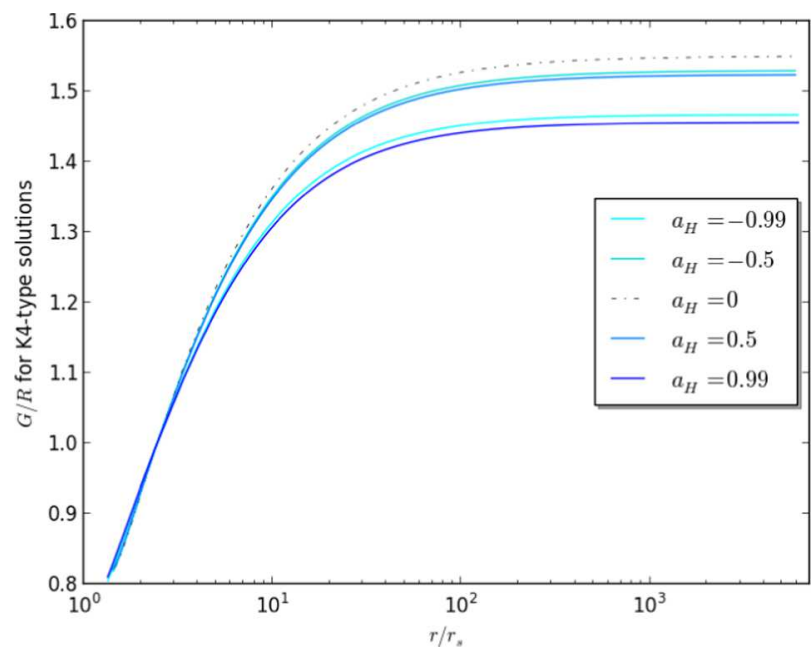

Fig. 19. Ratio of the cylindrical radius to the spherical radius for the conical K4-type solutions with a spin parameter $a_{H}$ varying between -0.99 and 0.99 vs. the distance $z$ above the equatorial plane in units of the Schwarzschild radius.

but the ratio of $G_{\infty} / G_{0}$ decreases only by a factor of 0.96 between a nonrotating and a maximally rotating black hole $\left(a_{H}=0.99\right)$. Hence, the faster the black hole rotates, the smaller the maximal jet radius. This result is expected because for a fast black hole rotation, the magnetic collimation efficiency parameter $(\epsilon)$ is higher.

The case of K3-type solutions is simple, as the factor $G$ increases with distance until it reaches a constant value. The ratio $G_{\infty} / G_{0}$ gives directly the expansion factor which is decreasing when $a_{H}$ increases from -0.99 up to 0.99 .

As can be seen from Fig. 18, the Lorentz factor maximum follows the opposite trend to $\epsilon$ for the conical K4-type solutions: the minimum value of $\epsilon$ is obtained for $a_{H} \simeq 0$ and for a nonrotating black hole the Lorentz factor curve reaches a maximum before decreasing up to a plateau at large distances. This type of curve is no longer observed when the absolute value of $a_{H}$ goes above some threshold. The increase of the plateau value for the Lorentz factor is much more pronounced for $a_{H}>0$ but can be seen also for negative values of the spin. At a distance of $r=1000 r_{s} \gamma$ increases from a value of the order of 6 for $a_{H}=0$ up to 14 for $a_{H}=0.99$.

For the conical solutions wherein the asymptotic geometry is fixed, their geometry is less affected. The effect of varying $\epsilon$ is different in this case, as compared to this effect for the cylindrically collimated solutions. To analyze the effect of collimation induced by the black hole spin, we plot the ratio of the cylindrical to spherical radius of the field lines for K4-type solutions and five different values of the black hole spin. We note that the ratio of the cylindrical to spherical radius of the field lines gives their opening angle with respect to the axis. The field lines start at the base of the jet with an opening angle which increases with the radial distance and expands away from the polar axis more rapidly for higher black hole rotation. The effect is more pronounced for negative spin parameters. At some distances above the equatorial plane $\left(z \simeq 100 r_{s}\right)$ the opening angle becomes constant as the field lines are finally becoming radial. It is found that for a given ratio of $\alpha / \alpha_{\text {lim }}$, where $\alpha_{\text {lim }}$ is the last open field line, the asymptotic opening angle is constant for all K4-type solutions, regardless of the spin of the black hole.

As seen in Fig. 19, the collimation of the jet increases with $\left|a_{H}\right|$ which is consistent with the increase of $\epsilon$. We note also that the base of the jet becomes slightly closer to the black hole horizon as $\left|a_{H}\right|$ increases.

Globally we see that the total geometry of the solution, and in particular the expansion of the jet radius, is extremely sensitive to the black hole speed.

\subsection{The magnetic flux and power of the jets}

Intuitively, by physical arguments of magnetic flux conservation, it is expected that magnetic fields not only play a dominant role in collimating large-scale AGN jets, but also critically affect the origin of the jets in accretion disks of black hole systems, which are accordingly termed magnetically arrested disks (Narayan et al. 2003). Indeed, theoretical modeling concludes that magnetic fields at the base of AGN jets are related to the corresponding accretion rate (Tchekhovskoy \& McKinney 2012). Zamaninasab et al. (2014) reported that the measured magnetic flux of the jet and the accretion disk luminosity are tightly correlated over several orders of magnitude for a sample of many radio-loud AGN, concluding thus that the jet-launching region is threaded by a dynamically important magnetic field. The magnetic fields of AGN can be measured either by the effect of a frequencydependent shift of the VLBI core position (known as the coreshift effect), or by Faraday rotation (e.g., Martí-Vidal et al. 2015, who reported magnetic fields of at least tens of Gauss on scales of the order of several light days -0.01 parsecs - from the black hole). Furthermore, MHD simulations in the frame of general relativity allow us to calculate the saturation or equilibrium value for the poloidal magnetic flux $\Phi_{\mathrm{BH}}$ threading the black hole (McKinney 2005, Tchekhovskoy et al. 2011, McKinney et al. 2012).

In Zamaninasab et al. (2014) this poloidal flux $\Phi_{\mathrm{BH}}$ is calculated as a function of the mass-accretion rate $\dot{M}$ as,

$\Phi_{\mathrm{BH}} \simeq 50 \sqrt{\dot{M} c\left(\frac{r_{s}}{2}\right)^{2}}$.

We consider that since such a strong magnetic flux can thread the black hole, we can use this formula to link our value of poloidal magnetic field at the Alfvén point $B_{\star}$ with the mass-accretion rate $\dot{M}$. In our model the magnetic flux from each hemisphere is given by $\Phi_{\mathrm{BH}}=\pi \varpi_{a}^{2} B_{\star}=\pi r_{\star}^{2} B_{\star} \alpha_{\text {lim. }}$. Then, the magnitude of the magnetic field is calculated from the expression

$B_{\star} \simeq 25 r_{s} \frac{\sqrt{\dot{M} c}}{\pi r_{\star}^{2} \alpha_{\lim }}=25 \mu \frac{\dot{M}^{1 / 2} c^{1 / 2}}{\pi r_{\star} \alpha_{\lim }}$.

In order to compare the jet power for the $\mathbf{K} \mathbf{2}$ and $\mathbf{K} \mathbf{3}$ solutions with the one obtained by general relativistic MHD simulations, we calculate this power in terms of the parameters of our model. Similarly to the way we deduced the angular momentum flux density in Eqs. (57) and (58), we may calculate the jet+counterjet power by substituting $\Psi_{A}$ from Eq. (56) and $\mathcal{E}=\mathcal{E}_{\star}\left(1+e_{1} \alpha\right)$ with $\mathcal{E}_{\star}$ from Eq. (60), and with the help of Eq. (40), we obtain, in terms of the constants of our model,

$$
\begin{aligned}
P_{\mathrm{jet}} & =\int_{0}^{A_{\mathrm{lim}}} \Psi_{A} \mathcal{E} \mathrm{d} A \\
& =\frac{v h_{\star}^{2} c}{2 \sqrt{\mu}}\left(B_{\star} r_{\star}\right)^{2} \int_{0}^{\alpha_{\mathrm{lim}}}\left(1+e_{1} \alpha\right) \sqrt{1+\delta \alpha} \mathrm{d} \alpha .
\end{aligned}
$$

Hence, we finally get

$P_{\text {jet }} \simeq 625 \frac{v \mu^{3 / 2} h_{\star}^{2}}{2 \pi^{2} \alpha_{\mathrm{lim}}^{2}} \dot{M} c^{2} \int_{0}^{\alpha_{\mathrm{lim}}}\left(1+e_{1} \alpha\right) \sqrt{1+\delta \alpha} \mathrm{d} \alpha$. 
Therefore, for the efficiency $\eta_{\text {jet }} \equiv P_{\text {jet }} / \dot{M} c^{2}$ for our $\mathbf{K} 2$ model we obtain a value $\eta_{\text {jet }} \simeq 0.52$, while for our $\mathbf{K} 3$ model we obtain $\eta_{\text {jet }} \simeq 0.40$. On the other hand, McKinney (2005) determined self-consistently the jet power in Blandford-Znajek numerical models and deduced an efficiency $\eta_{\text {jet }}$ between 0.01 and 0.1 for ultra-relativistic Poynting-dominated jets with $a_{H}$ larger than 0.8. Later, Tchekhovskoy et al. (2011) and McKinney et al. (2012) increased the magnetic flux which can be pushed near the black hole leading to magnetically arrested accretion and obtained values of the net flow efficiency larger than 1 for rapidly spinning black holes with $a_{H}$ larger than 0.9 . Their models that develop a highly non-axisymmetric magnetically choked accretion flow, initially have the poloidal component of the magnetic field dominant and the wind has an efficiency always smaller than that of the jet. We note that the net flow efficiency for the jet is equal to $\left(P_{\text {jet }}-\dot{M}_{H} c^{2}\right) /\left[\dot{M}_{H} c^{2}\right]_{t}$ where $\dot{M}_{H}$ is the black hole mass-accretion rate and $\left[\dot{M}_{H} c^{2}\right]_{t}$ is the time-averaged value of accretion power. This black hole massaccretion rate could be smaller than the mass-accretion rate measured by Zamaninasab et al. (2014). In fact, they deduced the accretion power by dividing the bolometric luminosity with a radiative efficiency of 0.4 . Larger values of the inflow rates $\dot{M}_{\text {in,i }}$ and $\dot{M}_{\text {in,o }}$ have been obtained by McKinney et al. (2012) at radii $5 r_{s}$ and $25 r_{s}$, respectively. Zdziarski et al. (2015) found also that the jet power moderately exceeds the accretion power $\dot{M} c^{2}$ for blazars estimating the magnetic flux from the radio-jet core-shift effect and the self-absorbed flux evaluation. However, there is a large scatter around the mean value for blazars $\eta_{\text {jet }} \simeq 1.3$ and the jet power for radio galaxies is smaller, especially for M 87. Therefore, our estimations of jet power from our K2 and K3 solutions with mass loading could perfectly match that for less-efficient blazars and radio galaxies. Moreover, they do not overly depend on the spin parameter $a_{H}$, since $\alpha_{\text {lim }}$ keeps a value slightly smaller than 1 when the spin parameter varies for the $\mathbf{K} \mathbf{3}$-type solutions, even for retrograde black holes.

At this point, we prefer to postpone a further discussion of the jet power, until we have completed our study, which also includes inflow solutions and leads to a spin-energy extraction or addition from the black hole (work in progress).

\section{Summary and conclusions}

As was pointed already in 1957 by Parker (see also Parker 1963), for the driving of the solar wind and similar enthalpydriven astrophysical outflows, some energy/momentum addition is required. The original isothermal and polytropic models with a heat conduction, have shown that effectively energy and/or momentum are necessary for producing supersonic/superAlfvenic outflows at large distances, to also meet the respective causality requirements. Quasi-radial wind-type astrophysical outflows with shock transitions (Habal \& Tsinganos 1983) have been applied to explain the appearance of emission knots in galactic (Silvestro et al. 1987) and extragalactic objects (Ferrari et al. 1984, Ferrari et al. 1986), also in the framework of special relativity (Ferrari et al. 1985).

When deviations of the outflow geometry from radial expansion exist and the problem is fully two-dimensional, these outflows can be collimated in the form of jets (Sauty \& Tsinganos 1994) mainly by magnetic fields with a suitable external gas pressure distribution. Along these lines, in Vlahakis \& Tsinganos (1998), the original Parker model was extended to include general MHD effects, in the context of meridional self-similarity. The present paper takes the extra step of using the framework of a Kerr metric to explore analogous enthalpy- or generalized pressure-driven outflows from the environment of a rotating black hole.

Specifically, in this paper we present an exact MHD solution for an outflow in a Kerr metric, constructed by using the assumption of self similarity and the mechanism for driving the outflow which is developed in Sauty \& Tsinganos (1994). Additionally, the model is based on a first-order expansion of the governing general relativistic equations in the magnetic flux function around the symmetry axis of the system. It yields four nonlinear and coupled differential equations as a function of the radius, for the Alfvén number, the gas pressure, the expansion function and the radius of the jet. The model depends on seven parameters. Two of them are the meridional increase of the gas pressure and the mass to magnetic flux ratio, $\kappa$ and $\delta$, respectively. There is also the meridional increase of the total energy with the magnetic flux function, $e_{1}$, the poloidal current density flowing along the system axis, $\lambda$, the escape speed in units of the Alfvén speed, $v$, the Schwarzschild radius in units of the Alfvén radius, $\mu$, and the dimensionless black hole spin $l$. In addition to those seven parameters, we have to adjust the pressure at the Alfvénic transition. We chose to adjust it such as to minimize the oscillations of the magnitude of the flow speed along the axis and taking the limiting solution. We also fix the magnetic field at the Alfvén transition, $B_{\star}$, and a uniform pressure constant $P_{0}$ to ensure a zero external heating at infinity along the axis where the Lorentz factor is maximum.

The model takes into account the light cylinder effects and the meridional increase of the Alfvén number with the magnetic flux function, $m_{1}$. This parameter is deduced from the regularity conditions at the Alfvén transition surface.

The classical energetic criterion for the transition from conical winds to cylindrical jets is generalized in general relativity and it amounts to say that if the total available energy along a nonpolar streamline exceeds the corresponding energy along the axis, then the outflow collimates in a jet.

In the framework of a Kerr metric, we illustrate the model with four different enthalpy-driven solutions wherein the contribution of the Poynting flux is rather small. The first three solutions are cylindrically collimated, while the fourth represents a conical outflow at infinity. The flow collimation is induced by electromagnetic forces. In all four models, relativistic speeds are obtained, while in one of them the Lorentz factor $\gamma$ obtains ultra-relativistic values. A preliminary application of one of our Kerr solutions (K3) was explored to model the spine jet in $\mathrm{M}$ 87, yielding encouraging results. A more complete modeling for the M 87 jet including an external disk-wind component will be explored in another study.

Our analytical solutions of the full general relativistic MHD equations in a Kerr metric may contribute to a better understanding of relativistic AGN jets and are complementary to sophisticated numerical simulations of such jets (e.g., McKinney 2005, Tchekhovskoy et al. 2011, McKinney et al. 2012). In both the analytical and the numerical approach, the outflows are electromagnetically confined. However, while in the above numerical simulations the outflow is driven electromagnetically (e.g., via the Blandford-Znajek mechanism), in the present analytical solutions the outflow from the hot corona surrounding the black hole is enthalpy- or generalized pressure-driven (e.g., via the Sauty-Tsinganos mechanism). Nevertheless, it is interesting to note that the jet powers for the two representative analytical solutions we present in this paper are similar to those determined by the numerical simulations. 
The present model can also serve to construct an inflow solution, in order to link it with an outflow solution and the physical creation of leptonic pairs to determine the energy balance of the black hole, via a generalized Penrose process as compared to the Blandford-Znajek mechanism. This undertaking is in progress and will be presented in another publication.

Acknowledgements. Part of this work was supported by the French Action Fédératrice CTA project and the "Programme Blanc" of Paris Observatory. The authors thank the referee for his constructive suggestions which lead to a better presentation of the results of the paper, in particular for a comparison with the results of numerical simulations.

\section{References}

Anderson, J. M., Li, Z.-Y., Krasnopolsky, R., \& Blandford, R. D. 2003, ApJ, 590, L107

Begelman, M. C., Fabian, A. C., \& Rees, M. J. 2008, MNRAS, 384, L19

Beskin, V. S. 2010, MHD Flows in Compact Astrophysical Objects: Accretion, Winds and Jets (Berlin, Heidelberg: Springer-Verlag)

Blandford, R. D., \& Znajek, R. L. 1977, MNRAS, 179, 433

Blandford, R. D., \& Payne, D. G. 1982, MNRAS, 199, 883

Böttcher, M., Reimer, A., Sweeney, K., \& Prakash, A. 2013, ApJ, 768, 54

Breitmoser, E., \& Camenzind, M. 2000, A\&A, 361, 207

Cayatte, V., Vlahakis, N., Matsakos, T., et al. 2014, ApJ, 788, L19

Casadio, C., et al. 2015, ApJ, 813, 51

Celotti, A., \& Ghisellini, G. 2008, MNRAS, 385, 283

Fabian, A. C., \& Rees, M. J. 1995, MNRAS, 277, L55

Feng, J., Wu, Q., \& Lu, R.-S. 2016, ApJ, 830, 6

Ferrari, A., Habbal, S. R., Rosner, R., \& Tsinganos, K. 1984, ApJ, 277, L35

Ferrari, A., Trussoni, E., Rosner, R., \& Tsinganos, K. 1985, ApJ, 294, 397

Ferrari, A., Trussoni, E., Rosner, R., \& Tsinganos, K. 1986, ApJ, 300, 577

Finke, J. D. 2016, ApJ, 830, 94

Giroletti, M., et al. 2004, ApJ, 600, 127

Ghisellini, G., Tavecchio, F., \& Chiaberge, M. 2005, A\&A, 432, 401

Globus, N., Sauty, C., Cayatte, V., \& Celnikier, L.-M. 2014, Phys. Rev. D, 89, 124015

Gracia, J., Vlahakis, N., Agudo, I., Tsinganos, K., \& Bogovalov, S. V. 2009, ApJ, 695,503

Hada, K., Doi, A., Kino, M., et al. 2011, Nature, 477, 185

Habal, S. R., \& Tsinganos, K., JGR, 88, 1965

Hervet, O., Meliani, Z., Zech, A., et al. 2017, A\&A, 606, A103

Komissarov, S. S. 2007, MNRAS, 382, 995

Kravchenko, E. V., Kovalev, Y. Y., Hovatta, T., \& Ramakrishnan, V. 2016, MNRAS, 462, 2747

Li, Y.-R., Yuan, Y.-F., Wang, J.-M., Wang, J.-C., \& Zhang, S. 2009, ApJ, 699, 513

Marscher, A. P., et al. 2010, ApJL, 710, 126

Martí, J. M., Perucho, M., \& Gómez, J. L. 2016, ApJ, 831, 163

Martí-Vidal, I., Muller, S., Vlemmings, W., Horellou, C., \& Aalto, S. 2015, Science, 348,311

McKinney, J. C. 2005, ApJ, 630, L5

McKinney, J. C., \& Blandford, R. D. 2009, MNRAS, 394, L126
McKinney, J. C., Tchekhovskoy, A., \& Blandford, R. D. 2012, MNRAS, 423, 3083

Meliani, Z., \& Keppens, R. 2010, A\&A, 520, L3

Meliani, Z., Sauty, C., Tsinganos, K., \& Vlahakis, N. 2004, A\&A, 425, 773

Meliani, Z., Sauty, C., Vlahakis, N., Tsinganos, K., \& Trussoni, E. 2006, A\&A, 447, 797

Meliani, Z., Sauty, C., Tsinganos, K., Trussoni, E., \& Cayatte, V. 2010, A\&A, 521, A67

Mertens, F., Lobanov, A. P., Walker, R. C., \& Hardee, P. E. 2016, A\&A, 595, A54

Mignone, A., Plewa, T., \& Bodo, G. 2005, ApJS, 160, 199

Nagai, H., et al. 2014, ApJ, 785, 53

Narayan, R., \& Yi, I., 1994, ApJ, 428, L13

Narayan, R., Igumenshchev, I. V., \& Abramowicz, M. A. 2003, PASJ, 55, L69

Nathanail, A., \& Contopoulos, I. 2014, ApJ, 788, 186

Parker, E. N. 1957, Phys. Rev., 107, 924

Parker, E. N. 1963, Interplanetary Dynamical Processes (New York: Interscience Publishers Division, John Wiley and Sons)

Petropoulou, M., \& Dermer, C. 2016, ApJ, 825, L11

Prieto, M. A., Fernández-Ontiveros, J. A., Markoff, S., Espada, D., \& GonzálezMartín, O. 2016, MNRAS, 457, 3801

Sauty, C., \& Tsinganos, K. 1994, A\&A, 287, 893

Sauty, C., Tsinganos, K., \& Trussoni, E. 1999, A\&A, 348, 327

Sauty, C., Trussoni, E., \& Tsinganos, K. 2002, A\&A, 389, 1068

Sauty, C., Trussoni, E., \& Tsinganos, K. 2004, A\&A, 421, 797

Sauty, C., Lima, J. J. G., Iro, N., \& Tsinganos, K. 2005, A\&A, 432, 687

Sikora, M. 2011, in Jets at all Scales, eds. G. Romero, R. Sunyaev, \& T. Bellon (Cambridge: Cambridge Univ. Press), IAU Symp. 275, 59

Sikora, M., Rutkowski, M., \& Begelman, M. C. 2016, MNRAS, 457, 1352

Silvestro, G., Ferrari, A., Rosner, R., Trussoni, E., \& Tsinganos, K. 1987, MNRAS, 325, 228

Sol, H., Pelletier, G., \& Asseo, E. 1989, MNRAS, 237, 411

Taub, A. H. 1948, PhRv, 74, 328

Tavecchio, F. 2016, AIP Conf. Proc., 1792, 020007

Tavecchio, F., \& Ghisellini, G. 2016, MNRAS, 456, 2374

Tchekhovskoy, A. 2015, in The Formation and Disruption of Black Hole Jets, eds. I. Contopoulos, D. Gabuzda, \& N. Kylafis, Astrophys. Space Sci. Lib., 414, 45

Tchekhovskoy, A., Narayan, R., \& McKinney, J. C. 2011, MNRAS, 418, L79

Tchekhovskoy, A., \& McKinney, J. C. 2012, MNRAS, 423, L55

Tsinganos, K. 1982, ApJ, 252, 775

Tsinganos, K., \& Trussoni, E. 1991, A\&A, 249, 156

Tsinganos, K., \& Sauty, C., 1992, A\&A, 255, 405

Urry, C. M., \& Padovani, P. 1995, PASP, 107, 803

van Eerten, H. J., Meliani, Z., Wijers, R. A. M. J., \& Keppens, R. 2011, MNRAS, 410, 2016

Vlahakis, N. 2015, in The Formation and Disruption of Black Hole Jets, eds I. Contopoulos, D. Gabuzda, \& N. Kylafis Astrophys. Space Sci. Lib. 414, 177

Vlahakis, N., \& Tsinganos, A. 1998, MNRAS, 298, 777

Vlahakis, N., \& Königl, A. 2003a, ApJ, 596, 1104

Vlahakis, N., \& Königl, A. 2003b, ApJ, 596, 1080

Vlahakis, N., \& Königl, A. 2004, ApJ, 605, 656

Vovk, I., \& Babić, A. 2015, A\&A, 578, A92

Zamaninasab, M., Clausen-Brown, E., Savolainen, T., \& Tchekhovskoy, A. 2014, Nature, 510, 126

Zdziarski, A. A., Sikora, M., Pjanka, P., \& Tchekhovskoy A. 2015, MNRAS, 451, 927 


\section{Appendix A: Kerr metric elements}

We can rewrite the Kerr-metric in a simpler form:

$\mathrm{d} s^{2}=-h^{2} c^{2} \mathrm{~d} t^{2}+h_{r}^{2} \mathrm{~d} r^{2}+h_{\theta}^{2} \mathrm{~d} \theta^{2}+h_{\phi}^{2}\left(\mathrm{~d} \phi+\beta^{\phi} c \mathrm{~d} t\right)^{2}$.

The Kerr metric elements are defined as

$h_{r}=\frac{\rho}{\sqrt{\Delta}}, h_{\theta}=\rho$,

$h_{\phi}=\varpi=\frac{\Sigma}{\rho} \sin \theta, \omega=\frac{\mathrm{car}_{s} r}{\Sigma^{2}}$,

$\beta_{\phi}=-\frac{\omega}{c} \varpi^{2}, \beta^{\phi}=-\frac{\omega}{c}$,

$h=\left(1-\frac{r_{s} r}{\rho^{2}}+\beta^{\phi} \beta_{\phi}\right)^{1 / 2}=\frac{\rho}{\Sigma} \sqrt{\Delta}$

Thus, to second order in $\sin \theta$ the metric is

$$
\begin{aligned}
\omega & =\frac{l c \mu R}{r_{\star}\left(R^{2}+l^{2}\right)^{2}}\left(1+\frac{l^{2} h_{z}^{2}}{R^{2}+l^{2}} \sin ^{2} \theta\right), \\
h & =\sqrt{1-\frac{\mu R}{R^{2}+l^{2}}}\left(1-\frac{\mu l^{2} R}{2\left(R^{2}+l^{2}\right)^{2}} \sin ^{2} \theta\right), \\
h_{r} & =\frac{1}{\sqrt{1-\frac{\mu R}{R^{2}+l^{2}}}}\left(1-\frac{l^{2}}{2\left(R^{2}+l^{2}\right)} \sin ^{2} \theta\right), \\
h_{\theta} & =r_{\star} \sqrt{R^{2}+l^{2}}\left(1-\frac{l^{2}}{2\left(R^{2}+l^{2}\right)} \sin ^{2} \theta\right), \\
h_{\phi} & =\varpi=r_{\star} \sqrt{R^{2}+l^{2}} \sin \theta .
\end{aligned}
$$

In order to simplify our notation, we define along the polar axis, the axial lapse function,

$h_{z}(R)=h_{r}^{-1}(R, \theta=0)=h(R, \theta=0)=\sqrt{1-\frac{\mu R}{R^{2}+l^{2}}}$,

and the axial shift of the metric,

$\omega_{z}(R)=\omega(R, \theta=0)=\frac{l c \mu R}{r_{\star}\left(R^{2}+l^{2}\right)^{2}}$

We can also write the metric as an expansion in $\alpha$ :

$$
\begin{aligned}
& \omega=\frac{l c \mu R}{r_{\star}\left(R^{2}+l^{2}\right)^{2}}\left(1+\frac{l^{2} h_{z}^{2} G^{2}}{\left(R^{2}+l^{2}\right)^{2}} \alpha\right), \\
& h=\sqrt{1-\frac{\mu R}{R^{2}+l^{2}}}\left(1-\frac{\mu l^{2} R G^{2}}{2\left(R^{2}+l^{2}\right)^{3}} \alpha\right), \\
& h_{r}=\frac{1}{\sqrt{1-\frac{\mu R}{R^{2}+l^{2}}}\left(1-\frac{l^{2} G^{2}}{2\left(R^{2}+l^{2}\right)^{2}} \alpha\right),} \\
& h_{\theta}=r_{\star} \sqrt{R^{2}+l^{2}}\left(1-\frac{l^{2} G^{2}}{2\left(R^{2}+l^{2}\right)^{2}} \alpha\right), \\
& h_{\phi}=\varpi=r_{\star} G \sqrt{\alpha} .
\end{aligned}
$$


L. Chantry et al.: Nonradial and nonpolytropic astrophysical outflows. X.

\section{Appendix B: Vectorial operators in Boyer-Lindquist coordinates of the Kerr metric.}

In this Appendix we summarize the expressions of the vectorial operators under the assumption of axisymmetry $\left(\partial_{\phi}=0\right)$.

1. The gradient vector $\nabla$ in the ZAMO orthonormal bases,

$\nabla=\sum_{i=1}^{3} \frac{\epsilon_{i}}{h_{i}} \partial_{i}$

2. The divergence of a vector $\boldsymbol{V}$,

$\nabla \cdot \boldsymbol{V}=\frac{1}{h_{r} h_{\theta} \varpi}\left[\partial_{r}\left(h_{\theta} \varpi V^{\hat{r}}\right)+\partial_{\theta}\left(h_{r} \varpi V^{\hat{\theta}}\right)\right]$

3. The scalar Laplace operator,

$\nabla^{2} A=\nabla \cdot \nabla A$

$$
=\frac{1}{h_{r} h_{\theta} \varpi}\left[\frac{1}{r_{\star}^{2}} \partial_{R}\left(\frac{h_{\theta} \varpi}{h_{r}} \partial_{R} A\right)+\partial_{\theta}\left(\frac{h_{r} \varpi}{h_{\theta}} \partial_{\theta} A\right)\right] .
$$

4. The curvature operator on a vector $\boldsymbol{V}$,

$\nabla \times \boldsymbol{V}=\frac{h_{k}}{h_{r} h_{\theta} h_{\phi}} \epsilon^{i j k} \partial_{i}\left(h_{j} V^{\hat{j}}\right) \boldsymbol{\epsilon}_{k}$,

$\nabla \times \boldsymbol{V}=\left[\begin{array}{c}\frac{1}{h_{\theta} \varpi} \partial_{\theta}\left(\varpi V^{\hat{\phi}}\right) \\ -\frac{1}{h_{r} \varpi r_{\star}} \partial_{R}\left(\varpi V^{\hat{\phi}}\right) \\ \frac{1}{h_{r} h_{\theta}}\left(\frac{1}{r_{\star}} \partial_{R}\left(h_{\theta} V^{\hat{\theta}}\right)-\partial_{\theta}\left(h_{r} V^{\hat{r}}\right)\right)\end{array}\right]$

5. The advection term,

$(\boldsymbol{V} \cdot \nabla) \boldsymbol{V}=V^{\alpha} D_{\alpha} V^{\beta} \boldsymbol{e}_{\beta}$

After some algebra we get for the poloidal component of the advection term:

$[(\boldsymbol{V} \cdot \nabla) \boldsymbol{V}]_{\mathrm{p}}=\left[\begin{array}{l}\frac{V^{\hat{r}}}{r_{\star} h_{r}} \partial_{R} V^{\hat{r}}+\frac{V^{\hat{\theta}}}{h_{\theta}} \partial_{\theta} V^{\hat{r}}+\frac{V^{\hat{r}} V^{\hat{\theta}}}{h_{\theta}} \partial_{\theta} \ln \left(h_{r}\right)-\frac{\left(V^{\hat{\theta}}\right)^{2}}{h_{r}} \partial_{r} \ln \left(h_{\theta}\right)-\frac{\left(V^{\hat{\phi}}\right)^{2}}{h_{r}} \partial_{r} \ln \left(h_{\phi}\right) \\ \frac{V_{\star} h_{r}}{r_{\star}} \partial_{R} V^{\hat{\theta}}+\frac{V^{\hat{\theta}}}{h_{\theta}} \partial_{\theta} V^{\hat{\theta}}+\frac{V^{\hat{r}} V^{\hat{\theta}}}{r_{\star} h_{r}} \partial_{R} \ln \left(h_{\theta}\right)-\frac{\left(V^{\hat{r}}\right)^{2}}{h_{\theta}} \partial_{\theta} \ln \left(h_{r}\right)-\frac{\left(V^{\phi}\right)^{2}}{h_{\theta}} \partial_{\theta} \ln \left(h_{\phi}\right)\end{array}\right]$.

It can be useful to get the non-symmetric advection term,

$[(\boldsymbol{B} \cdot \nabla) \boldsymbol{C}]=\left[\begin{array}{c}\frac{B^{\hat{r}}}{r_{\star} h_{r}} \partial_{R} C^{\hat{r}}+\frac{B^{\hat{\theta}}}{h_{\theta}} \partial_{\theta} C^{\hat{r}}+\frac{B^{\hat{r}} C^{\hat{\theta}}}{h_{\theta}} \partial_{\theta} \ln \left(h_{r}\right)-\frac{B^{\hat{\theta}} C^{\hat{\theta}}}{h_{r}} \partial_{r} \ln \left(h_{\theta}\right)-\frac{B^{\hat{\phi}} C^{\hat{\phi}}}{h_{r}} \partial_{r} \ln \left(h_{\phi}\right) \\ \frac{B_{\star} h_{r}}{r_{R}} \partial_{R} C^{\hat{\theta}}+\frac{B^{\theta}}{h_{\theta}} \partial_{\theta} C^{\hat{\theta}}+\frac{C^{\hat{r}} B^{\hat{\theta}}}{r_{\star} h_{r}} \partial_{R} \ln \left(h_{\theta}\right)-\frac{B^{\hat{C}} h^{\hat{r}}}{h_{\theta}} \partial_{\theta} \ln \left(h_{r}\right)-\frac{B^{\hat{\phi}} C^{\hat{\phi}}}{h_{\theta}} \partial_{\theta} \ln \left(h_{\phi}\right) \\ \frac{B^{\hat{r}}}{h_{r} r_{\star}} \partial_{R} C^{\hat{\phi}}+\frac{B^{\hat{\theta}}}{h_{\theta}} \partial_{\theta} C^{\hat{\phi}}+\frac{C^{\hat{r}} B^{\hat{\phi}}}{r_{\star} h_{r}} \partial_{R} \ln \left(h_{\phi}\right)+\frac{C^{\hat{\theta}} B^{\hat{\phi}}}{h_{\theta}} \partial_{R} \ln \left(h_{\phi}\right)\end{array}\right]$.

\section{Appendix C: Final differential equations of the MHD problem}

The final ordinary differential equations of our model can be written as :

$R \frac{\mathrm{d}}{\mathrm{d} R}\left(\begin{array}{c}M^{2} \\ F\end{array}\right)=\frac{1}{\mathcal{D}\left(M^{2}, G^{2}, F, R\right)}\left(\begin{array}{c}\mathcal{N}_{M^{2}} \\ \mathcal{N}_{F}\end{array}\right)$

where

$$
\mathcal{D}\left(m^{2}, G^{2}, F, R\right)=\frac{h_{\star}^{2}}{R}\left[-D\left(1+\left(\kappa-2 m_{1}\right) \frac{R^{2}+l^{2}}{G^{2}}-\frac{l^{2}}{R^{2}+l^{2}}\right)+\frac{\lambda^{2} \Lambda^{2} N_{B}^{2}\left(R^{2}+l^{2}\right)}{D^{2}}+\frac{h_{z}^{4} F^{2}\left(R^{2}+l^{2}\right)}{4 h_{\star}^{2} R^{2}}\right],
$$


and

$$
\begin{aligned}
\mathcal{N}_{F}= & \frac{F M^{2}}{h_{\star}^{2}}\left[\frac{F}{2}\left(\frac{h_{z}^{2} F}{2}-1\right)+\left(\frac{F}{2}-1\right)\left(1+\left(\kappa-2 m_{1}\right) \frac{X_{+}}{G^{2}}-\frac{l^{2}}{X_{+}}+\frac{\lambda^{2} \Lambda^{2} N_{B}^{2} X_{+}}{D^{3}}\right)\right] \\
& +\frac{R^{2} h_{z}^{2}}{X_{+} h_{\star}^{2}}\left[\frac{X_{+}}{R^{2}} F(F-1)-\frac{2}{h_{z}^{2}}-\frac{4 \lambda^{2} \mu h_{\star}^{2} \Lambda^{2} X_{+}}{v^{2} h_{z}^{4}}-\frac{4 l^{2} \mu R}{h_{z}^{2} X_{+}^{2}}\right]\left[1+\left(\kappa-2 m_{1}\right) \frac{X_{+}}{G^{2}}-\frac{l^{2}}{X_{+}}-\frac{\lambda^{2} \Lambda^{2} N_{B}^{2} X_{+}}{D^{3}}-\frac{h_{z}^{2} F^{2} X_{+}}{4 R^{2}}\right] \\
& +\left(\frac{2 \kappa \Pi G^{2} R^{2}}{h_{\star}^{2}}+\frac{\mu F R X_{-}}{h_{\star}^{2} X_{+}^{2}}\right)\left[1+\left(\kappa-2 m_{1}\right) \frac{X_{+}}{G^{2}}-\frac{l^{2}}{X_{+}}-\frac{\lambda^{2} \Lambda^{2} N_{B}^{2} X_{+}}{D^{3}}-\frac{h_{z}^{2} F^{2} X_{+}}{4 R^{2}}\right] \\
& +\frac{v^{2} h_{\star}^{2} F G^{2} R}{2 h_{z}^{2} M^{2}} \frac{X_{-}}{X_{+}}\left(\kappa-2 e_{1}+2 m_{1}-\delta\right)-\frac{\mu F M^{2} R X_{-}}{2 h_{\star}^{2} h_{z}^{2} X_{+}^{2}}\left[1+\left(\kappa-2 m_{1}\right) \frac{X_{+}}{G^{2}}-\frac{l^{2}}{X_{+}}\right] \\
& +\frac{\lambda^{2} \mu \Lambda^{2} N_{B} N_{V} F R}{h_{\star}^{2} D^{3}} \frac{X_{-}}{X_{+}}-\frac{\lambda^{2} \Lambda N_{B} h_{z}^{2} F X_{+}}{h_{\star}^{2} D^{2}}\left(F-\frac{2 R^{2}}{X_{+}}\right)+\frac{\lambda^{2} \mu h_{\star}^{2} F G^{2}}{h_{z}^{2} M^{2}} \frac{R X_{-}}{X_{+}}\left(\frac{\Lambda^{2} N_{B}}{D}+\frac{\bar{\omega}_{z}}{\lambda}\right) \\
& +\frac{4 \lambda^{2} \Lambda^{2} R^{2}}{h_{z}^{2}}\left(\frac{N_{B}^{2}}{D^{2}}-\frac{h_{z}^{2}}{2 M^{2}} \frac{N_{V}^{2}}{D^{2}}\right)\left[1+\left(\kappa-2 m_{1}\right) \frac{X_{+}}{G^{2}}-\frac{l^{2}}{X_{+}}-\frac{\lambda^{2} \Lambda^{2} N_{B}^{2} X_{+}}{D^{3}}-\frac{h_{z}^{2} F}{2}\right] \\
& +\lambda \sqrt{\mu} v l h_{\star} \frac{\Lambda G^{2} F R}{D} \frac{\left(3 R^{2}-l^{2}\right)}{X_{+}^{2}}\left(\frac{N_{B}}{h_{\star}^{2} D}-\frac{N_{V}}{M^{2}}\right) \\
& -\frac{2 v^{2} l^{2} h_{\star}^{2} G^{4}}{h_{z}^{2} M^{2}} \frac{R^{3}}{X_{+}^{3}}\left[1-\frac{l^{2}}{X_{+}}+\left(\kappa-2 m_{1}\right) \frac{X_{+}}{G^{2}}-\frac{\lambda^{2} \Lambda^{2} N_{B}^{2} X_{+}}{D^{3}}+\left(\frac{X_{-}}{4 R^{2}}+\frac{h_{z}^{2}}{2}\right) F\right] \\
& +\frac{2 M^{2} l^{2} R^{2}}{h_{\star}^{2} h_{z}^{2} X_{+}^{2}}\left[1+\left(\kappa-2 m_{1}\right) \frac{X_{+}}{G^{2}}-\frac{l^{2}}{X_{+}}-\frac{\lambda^{2} \Lambda^{2} N_{B}^{2} X_{+}}{D^{3}}+\frac{h_{z}^{2} F X_{+}}{R^{2}}\left(\frac{3 R^{2}}{2 X_{+}}+\left(\kappa-2 m_{1}\right) \frac{X_{+}}{G^{2}}\right)\right],
\end{aligned}
$$

where $X_{+}=R^{2}+\ell^{2} \quad X_{-}=R^{2}-\ell^{2}$.

$$
\begin{aligned}
& \mathcal{N}_{M^{2}}= \frac{M^{4}}{4 h_{\star}^{2}}\left[-h_{z}^{2} F^{2}+2 F-\frac{4 R^{2} l^{2}}{X_{+}^{2}}-4\left(F-2 \frac{R^{2}}{X_{+}}\right)\left(1+\left(\kappa-2 m_{1}\right) \frac{X_{+}}{G^{2}}-\frac{l^{2}}{X_{+}}\right)\right] \\
&+\frac{h_{z}^{2} M^{2}}{h_{\star}^{2}}\left[\frac{h_{z}^{2} X_{+} F^{3}}{8 R^{2}}+\frac{h_{z}^{2} F^{2}}{4}\left(1+\frac{\mu X_{-}}{h_{z}^{2} R X_{+}}\right)+\left(\kappa-2 m_{1}\right) \frac{X_{+} F}{G^{2}}-F \frac{\lambda^{2} \mu}{v^{2}} X_{+} \Lambda^{2} \frac{h_{\star}^{2}}{h_{z}^{2}}-2 \frac{R^{2}}{X_{+}}-\left(\kappa-2 m_{1}\right) \frac{2 R^{2}}{G^{2}}+\frac{3 R^{2} l^{2}}{X_{+}^{2}}-\frac{F l^{2}}{X_{+}}\left(\frac{3}{2}-h_{z}^{2}\right)\right] \\
&+\frac{v^{2} h_{\star}^{4} D R G^{2}}{2 h_{z}^{2} M^{2}} \frac{X_{-}}{X_{+}}\left(\kappa-\delta+2 m_{1}-2 e_{1}\right)+\kappa \frac{X_{+}}{2} \frac{h_{z}^{2}}{h_{\star}^{2}} F \Pi G^{2} M^{2}-\frac{D M^{2} \mu R X_{-}}{2 h_{z}^{2} X_{+}^{2}}\left[1+\left(\kappa-2 m_{1}\right) \frac{X_{+}}{G^{2}}-\frac{l^{2}}{X_{+}}\right]+\frac{\lambda^{2} \mu R \Lambda^{2} N_{B} N_{V}}{D^{2}} \frac{X_{-}}{X_{+}} \\
&+\lambda^{2} \Lambda^{2} X_{+}\left(\frac{N_{B}^{2}}{D^{2}}-\frac{h_{z}^{2}}{2 M^{2}} \frac{N_{V}^{2}}{D^{2}}\right)\left(2 M^{2} \frac{R^{2}}{X_{+}}+h_{z}^{2}\left(F-2 \frac{R^{2}}{X_{+}}\right)\right)-\frac{\lambda^{2} \Lambda N_{B} h_{z}^{2} X_{+}}{D}\left(F-2 \frac{R^{2}}{X_{+}}\right)+\frac{\lambda^{2} \mu h_{\star}^{4} R G^{2}}{h_{z}^{2} M^{2}} \frac{X_{-}}{X_{+}}\left(\Lambda^{2} N_{B}+\frac{D r_{\star} \omega_{z}}{\lambda V_{\star} h_{\star}}\right) \\
&-\frac{l^{2} v^{2} R G^{4} h_{\star}^{2}}{2 M^{2} X_{+}^{3}}\left[h_{\star}^{2} D\left(3 R^{2}-l^{2}+\frac{\mu R X_{-}}{h_{z}^{2} X_{+}}\right)+h_{z}^{2} F X_{+}\right]+\lambda \sqrt{\mu} v l h_{\star} R G^{2} \Lambda \frac{l^{2}-3 R^{2}}{X_{+}^{2}}\left(\frac{N_{V} h_{\star}^{2}}{M^{2}}-\frac{N_{B}}{D}\right), \\
& \frac{\mathrm{d} \Pi}{\mathrm{d} R}=- \frac{2}{h_{z}^{2} G^{4}}\left[\frac{\mathrm{d}}{\mathrm{d} R} M^{2}+\frac{M^{2}}{R}\left(F-\frac{2 R^{2}}{R^{2}+l^{2}}\right)\right]-\frac{1}{h_{z}^{4} M^{2}} \frac{R^{2}-l^{2}}{\left(R^{2}+l^{2}\right)^{2}}\left(v^{2} h_{\star}^{4}-\frac{\mu M^{4}}{G^{4}}\right) \\
& \frac{\mathrm{d} G^{2}}{\mathrm{~d} R}=\frac{G^{2}}{R}\left(\frac{2 R^{2}}{R^{2}+l^{2}}-F\right) .
\end{aligned}
$$

